ARTICLE

https://doi.org/10.1038/s41467-020-19068-2

\title{
Akt1 and dClZ1 promote cell survival from apoptotic caspase activation during regeneration and oncogenic overgrowth
}

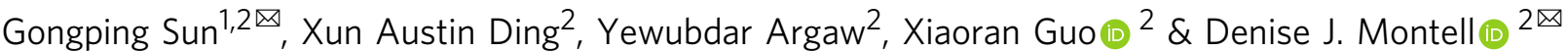

\begin{abstract}
Apoptosis is an ancient and evolutionarily conserved cell suicide program. During apoptosis, executioner caspase enzyme activation has been considered a point of no return. However, emerging evidence suggests that some cells can survive caspase activation following exposure to apoptosis-inducing stresses, raising questions as to the physiological significance and underlying molecular mechanisms of this unexpected phenomenon. Here, we show that, following severe tissue injury, Drosophila wing disc cells that survive executioner caspase activation contribute to tissue regeneration. Through RNAi screening, we identify akt 1 and a previously uncharacterized Drosophila gene CG8108, which is homologous to the human gene $\mathrm{CIZ1}$, as essential for survival from the executioner caspase activation. We also show that cells expressing activated oncogenes experience apoptotic caspase activation, and that Akt1 and $\mathrm{dClZ1}$ are required for their survival and overgrowth. Thus, survival following executioner caspase activation is a normal tissue repair mechanism usurped to promote oncogene-driven overgrowth.
\end{abstract}

\footnotetext{
${ }^{1}$ The Key Laboratory of Experimental Teratology, Ministry of Education and Department of Anatomy and Histoembryology, School of Basic Medical Sciences, Cheeloo College of Medicine, Shandong University, Jinan, Shandong, China. ${ }^{2}$ Molecular, Cellular, and Developmental Biology Department, University of

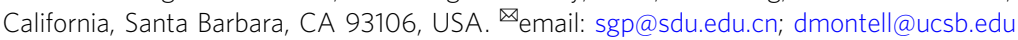


recise control of cell death and survival is critical for tissue homeostasis. Apoptosis is an important form of programmed cell death executed by proteolytic enzymes called caspases. While physiological apoptosis promotes normal development and homeostasis, inappropriate apoptosis causes tissue damage, degenerative diseases, and immune system dysfunction ${ }^{1}$.

In the process of apoptosis, activation of executioner caspases results in cleavage of hundreds of proteins, thereby dismantling the cell. Executioner caspase activation has thus been considered a point of no return ${ }^{2}$. However, recent studies show that some cells can survive apoptosis-inducing stresses even after executioner caspase activation ${ }^{3-8}$. Cultured mammalian cells can recover from executioner caspase activation induced by transient exposure to apoptotic inducers like ethanol, staurosporine, and $\mathrm{TNF}$, through a process called anastasis ${ }^{3,4,6}$. Similar events have been described in tumors cells after tBid overexpression ${ }^{5}$ or treatment with chemotherapeutic drugs like paclitaxel ${ }^{8}$. Survival after stress-induced executioner caspase activation has also been reported in cardiomyocytes after ischemia-reperfusion ${ }^{9}$ and in neurons expressing pathogenic tau ${ }^{10}$ in mice. These studies suggest that, in addition to the established competition between pro-death and pro-survival signals upstream of caspases, there is another layer of regulation after executioner caspases activation (Fig. 1a). Deciphering these regulatory mechanisms could therefore identify new therapeutic targets for a variety of diseases caused by improper cell death versus survival decisions.

To identify cells that survive executioner caspase activation in vivo, we previously developed a biosensor, CasExpress, which labels cells that have experienced transient caspase activation in Drosophila (Fig. 1b) ${ }^{11}$. We discovered widespread survival after executioner caspase activation during normal development of Drosophila larval and adult tissues including rapidly proliferating larval imaginal discs ${ }^{11}$. A similar sensor exhibits similar activation in adult tissues ${ }^{12}$. Here, we use the CasExpress sensor to decipher the physiological importance of, and molecular mechanisms enabling, survival from stress-induced executioner caspase activation in vivo. We show that although executioner caspase activation induced by heat shock, X-ray irradiation, or transient proapoptotic gene overexpression causes extensive cell death in wing imaginal discs, many epithelial cells survive and participate in tissue regeneration. We also report that wing disc epithelial cells experience transient apoptosis-inducing stresses even during normal development. To investigate the molecular mechanisms enabling cell survival from transient caspase activation, we developed a second-generation CasExpress sensor compatible with RNAi screening. We identified Aktl and a new gene, CG8108 (dCIZ1), as essential for survival from executioner caspase activation. Finally, we show that cells expressing activated oncogenes experience homeostatic apoptotic stress and exploit Akt1 and dCIZ1 to survive. Thus, survival from executioner caspase activation is a normal cellular response to tissue injury that promotes repair and can be hijacked to drive tumor growth.

\section{Results}

Survival from heat shock- or X-irradiation-induced executioner caspase activation contributes to tissue regeneration. The observations that a variety of cells can survive executioner caspase activation in response to diverse stresses (Fig. 1a) raises the question as to the physiological significance of this unexpected cellular resilience. We hypothesized that it might represent an injury repair mechanism. To test whether cells can survive executioner caspase activation after tissue damage in vivo, we exposed Drosophila larvae to heat shock and radiation, which are known to cause massive apoptosis in wing imaginal discs ${ }^{13}$. To assess whether any cells survived executioner caspase activation, we took advantage of the CasExpress biosensor ${ }^{11}$. CasExpress is a fusion protein composed of the extracellular and transmembrane domains of $\mathrm{mCD} 8,200$ amino acids from an endogenous executioner caspase target protein containing the proteolytic cleavage site (DQVD), and the transcription factor Gal4 (Fig. 1b). Upon caspase cleavage, Gal4 is released and translocates to the nucleus where, in combination with the G-trace lineage tracing system $^{14}$, it catalyzes DNA recombination that results in permanent GFP expression in that cell and all of its progeny ${ }^{11}$. In the presence of Gal $80^{\text {ts }}$, Gal4 activity is repressed at $18^{\circ} \mathrm{C}$. We grew animals at $18^{\circ} \mathrm{C}$ to the mid-third instar larval stage to silence reporter activity during normal development. Larvae were then exposed to $2 \mathrm{hrs}$ heat shock at $37^{\circ} \mathrm{C}$ or $10 \mathrm{~Gy} \mathrm{X}$-ray and transferred to $29^{\circ} \mathrm{C}$ to allow Gal4 to function (Fig. 1c). After $24 \mathrm{~h}$ at $29^{\circ} \mathrm{C}$, we stained for cleaved Dcp- 1 to detect dead cells. We note that cleaved Dcp-1 (cDcp1), which is an active form of the executioner caspase Dcp-1, accumulates detectably only in dead cells. In living/recovering cells, active caspase turns over rapidly and does not accumulate ${ }^{15}$. Moreover, dead/dying cells shrink, further concentrating the signal in small, intense clusters. Dead cells are also extruded and thus are primarily located in a different focal plane from the living epithelium. We confirmed that extensive apoptotic cell death occurs in the discs of irradiated or heat-shocked larvae ${ }^{13}$ (Supplementary Figure S1a-c).

To determine whether any cells that activated executioner caspase survived, we examined CasExpress flies for GFP expression. One day after stress, $\sim 20 \%$ of cells in discs from heat-shocked larvae and $\sim 30 \%$ of cells in the discs from irradiated animals were $\mathrm{GFP}^{+}\left(\right.$CasExpress $\left.^{+}\right)$, compared to $0.9 \%$ in the mock-treated controls (Fig. 1d-f, m). Discs expressing a caspaseinsensitive control sensor, in which the executioner caspase cleavage site DQVD is mutated to DQVA, showed no GFP ${ }^{+}$cells after stress (Supplementary Figure S1d-f). The stress-induced CasExpress activation was blocked in discs that lacked the initiator caspase Dronc (Supplementary Figure S1g-i). We then tested whether caspase activation in the $\mathrm{GFP}^{+}$cells required the pro-apoptotic proteins Reaper (Rpr), Head involution defective (Hid), and Grim (Fig. 1a). Drosophila lacking rpr, hid, and grim die as embryos ${ }^{16}$, precluding analysis of triple mutant larvae. Animals homozygous for rpr and grim and heterozygous for hid are viable to larval stages and exhibited a significant reduction in the percentage of $\mathrm{GFP}^{+}$cells after stress (Fig. $1 \mathrm{~g}-\mathrm{i}, \mathrm{m}$ ), indicating that CasExpress activation depends on these initiators of apoptosis.

To determine whether the $\mathrm{GFP}^{+}$cells in the stressed discs ultimately contributed to the regenerated discs, after stress and one day at $29^{\circ} \mathrm{C}$, we transferred the larvae back to $18^{\circ} \mathrm{C}$ for an additional 3 days recovery (Fig. 1c). At the time of dissection, all $\mathrm{GFP}^{+}$cells should be the progeny of cells that activated executioner caspase during the one day at $29^{\circ} \mathrm{C}$. Four days after stress, the discs exhibited normal morphology (Supplementary Figures $\mathrm{S} 1 \mathrm{j}-\mathrm{l}$ ), and the $\mathrm{cDcpl}^{+}$dead cells in the discs were diminished (compare Supplementary Figures S1m-o to Supplementary Figures $\mathrm{S} 1 \mathrm{a}-\mathrm{c}$ ), indicating the discs had regenerated. Importantly, a large proportion of the regenerated discs were $\mathrm{GFP}^{+}$(Fig. $\left.1 \mathrm{j}-\mathrm{m}\right)$, and some of the $\mathrm{GFP}^{+}$cells were proliferating, demonstrated by co-localization of GFP and phospho-histone H3 (PH3) staining (Supplementary Figure S1p). Therefore, we conclude that cells that survived stress-induced executioner caspase activation contributed to tissue regeneration following injury.

To determine whether cells that survived stress-induced executioner caspase activation were capable of differentiating, we examined regenerated eye discs after radiation. In early larval stages, like wing discs, eye disc cells are proliferative. At the beginning of the third instar, cells begin to differentiate into 
b
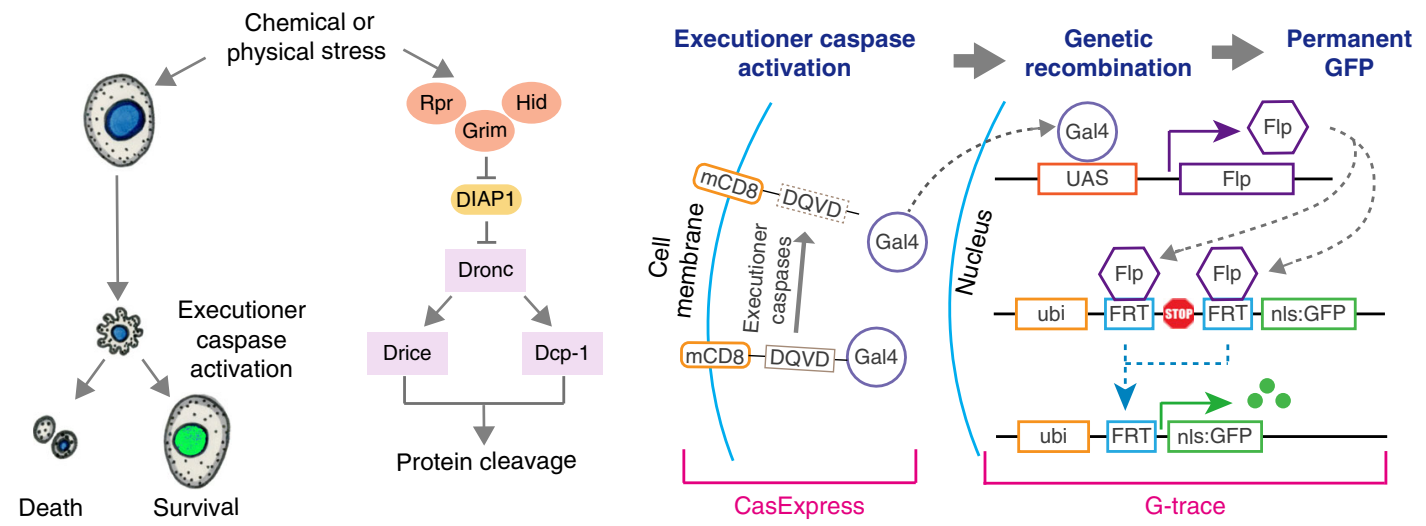

c

CasExpress $>$ G-trace Gal80ts

\section{$8^{\circ} \mathrm{C}$}

$1 \longleftarrow$ Days post stress (dps) $\longrightarrow \mathbf{I}$
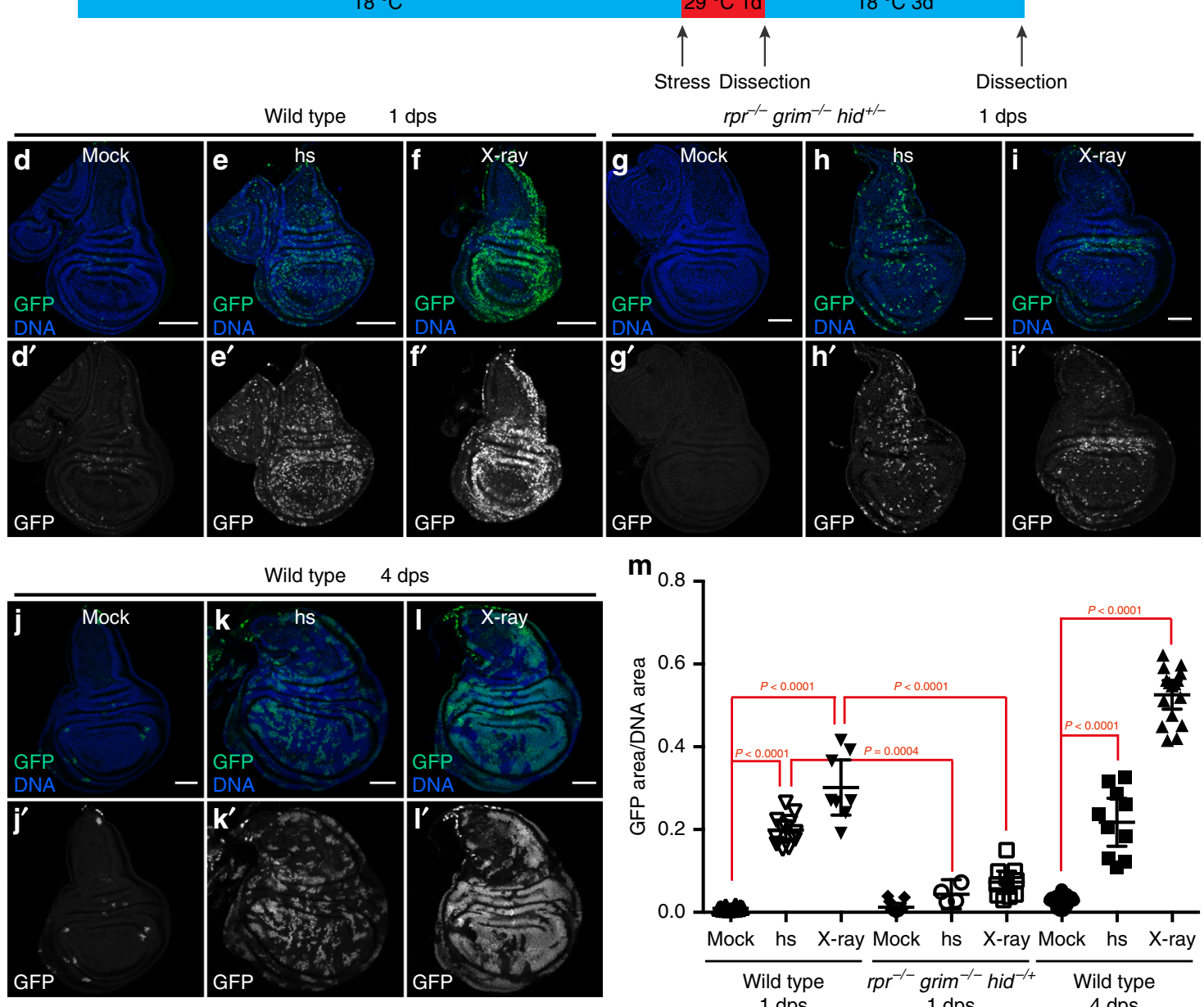

m

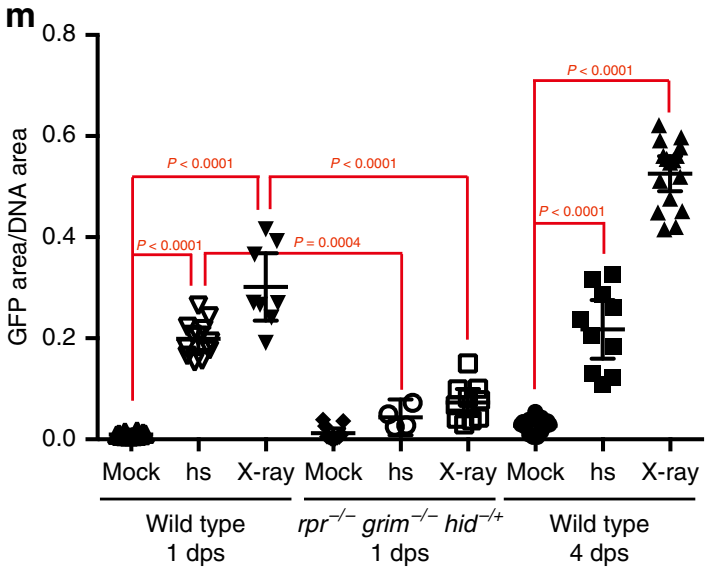

Fig. 1 Survival from executioner caspase activation contributes to regeneration after heat shock or $\mathbf{X}$-ray irradiation. (a) A schematic representation of cellular and molecular events in cells that experience stress-induced executioner caspase activation. (b) A schematic of CasExpress and G-trace. (c) A schematic of the experimental design for results shown in (d-m). (d-f) CasExpress activation (GFP) in wild type wing discs after mock treatment (d), heat shock (hs) (e), or radiation (X-ray) (f) and $1 \mathrm{~d}$ at $29^{\circ} \mathrm{C}$. (g-i) CasExpress activation (GFP) in rpr ${ }^{-/-}$grim $^{-/-}$hid $^{-/+}$wing discs after mock treatment (g), heat shock $(\mathbf{h})$, or radiation $(\mathbf{i})$ and $1 \mathrm{~d}$ at $29^{\circ} \mathrm{C}$. (j-I) CasExpress activation (GFP) in wild type wing discs after mock treatment $(\mathbf{j})$, heat shock $(\mathbf{k})$, or radiation (I), $1 \mathrm{~d}$ at $29^{\circ} \mathrm{C}$, and $3 \mathrm{~d}$ at $18^{\circ} \mathrm{C}$. All scale bars represent $50 \mu \mathrm{m}$. (m) Quantification of the percentage of GFP + cells in discs in (d-I). $n=12($ wild

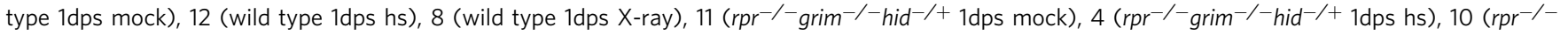
grim $^{-/- \text {hid }^{-/+}}$1dps X-ray), 13 (wild type 4dps mock), 10 (wild type 4dps hs), 16 (wild type $4 \mathrm{dps} X$-ray). $n$ is the number of biological independent samples used for quantification. The data are presented as mean values $\pm 95 \%$ confidence interval. Statistical significance was determined after the logarithm transformation using one-way ANOVA. The Tukey test was used to derive adjusted $P$-value for multiple comparisons. Source data are provided as a Source Data file. 
multiple cell types including photoreceptor neurons. We irradiated larvae carrying CasExpress, G-trace, and Galsots , and transferred them to $29^{\circ} \mathrm{C}$ for 1day to allow Gal4 to function prior to the initiation of differentiation, and then returned them to $18^{\circ} \mathrm{C}$ (Supplementary Figure S1q). After 4 days at $18^{\circ} \mathrm{C}$, we found a large proportion of differentiated neurons $\left(\mathrm{elav}^{+}\right)$were $\mathrm{GFP}^{+}$cells (Supplementary Figure S1s), indicating that cells that survived X-ray-induced executioner caspase activation can differentiate normally.

Cells can survive rpr-induced executioner caspase activation. To test whether cells can recover from more direct activation of the apoptotic pathway caused by overexpression of the proapoptotic protein Rpr, we used the LexA/lexO transcription system to drive rpr specifically in the central spalt (sal) domain of the wing pouch (Fig. 2a). We used a Gal80-regulatable version of LexA, LHG, and Gal80 $0^{\text {ts }}$, to control the timing of rpr overexpression $^{17,18}$ (Fig. 2a). As expected, discs overexpressing

a

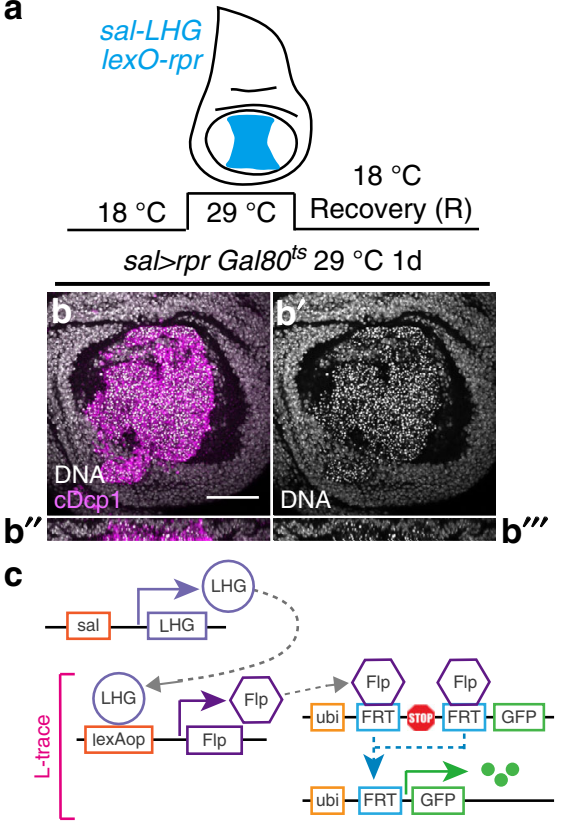

salsL-trace Gal80ts $29^{\circ} \mathrm{C} 1 \mathrm{~d}$ R3d

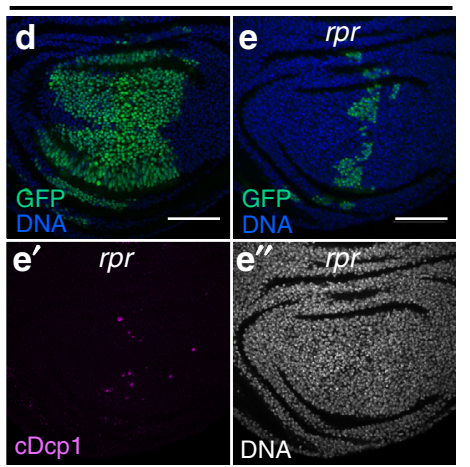

$r p r$ for one day exhibited intense executioner caspase activation and cell death, as shown by accumulation of cells with cDcpl and pyknotic nuclei (Fig. 2b-b"'). To test if any rpr-overexpressing cells survived, we used L-trace (Fig. 2c) which, similar to G-trace, permanently labels cells that originate from the sal-LHGexpressing region with GFP. After $r p r$ overexpression for one day at $29^{\circ} \mathrm{C}$, larvae were transferred back to $18^{\circ} \mathrm{C}$ for recovery. Three days later, the dead cells had been mostly eliminated and normal disc morphology restored (Fig. 2e-e"). A large fraction of the rproverexpressing cells died and were eliminated from the epithelium (compare Fig. 2e to d). However, the presence of GFP ${ }^{+}$cells in the restored epithelium (Fig. 2e) indicated that a fraction of cells can survive transient $r p r$ overexpression.

To assess whether these survivors had experienced executioner caspase activation or had simply escaped either $r p r$ expression or caspase activation, we used CasExpress and G-trace to monitor survival from executioner caspase activation following $r p r$ overexpression (Fig. 2f). Compared to controls in which $r p r$

f
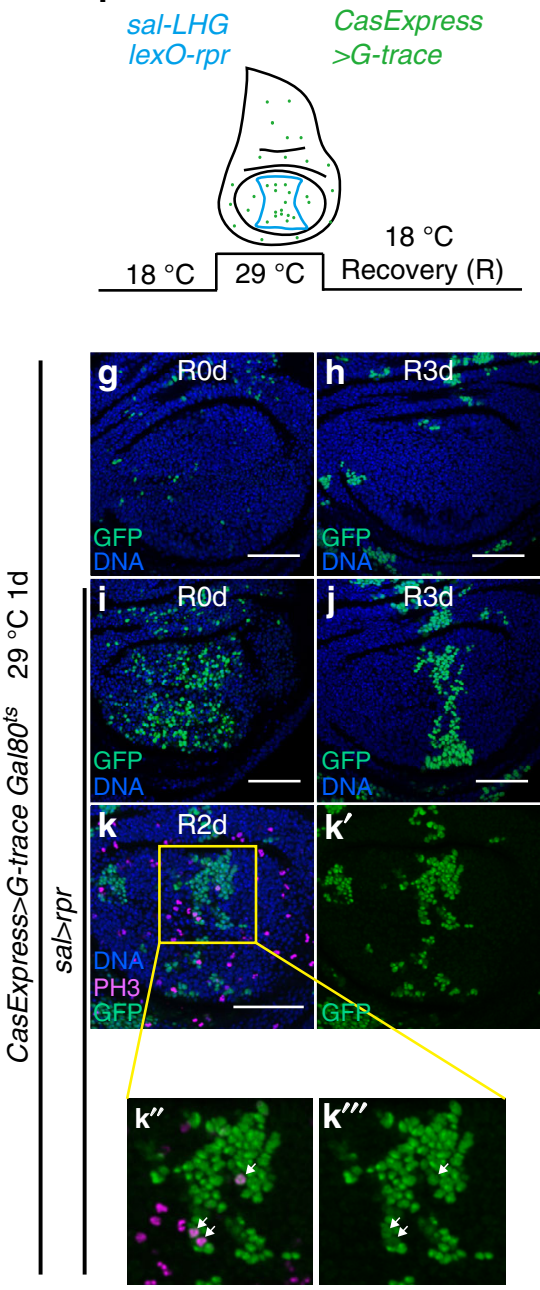

Fig. 2 Cells can survive rpr-induced executioner caspase activation. (a) A schematic showing the spalt (sal) domain (blue region) and the design for experiments in (b, d, and e). Transgenes were expressed only at $29^{\circ} \mathrm{C}$. (b) Overexpression of $r p r$ for $1 \mathrm{~d}$ induced extensive apoptotic cell death

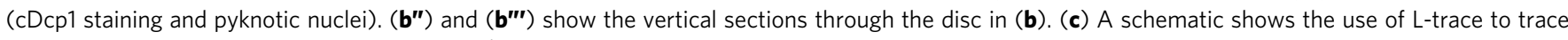
LHG-expressing cells. (d) sal-LHG L-trace Gal80ts disc after $1 \mathrm{~d}$ at $29^{\circ} \mathrm{C}$ and $3 \mathrm{~d}$ at $18^{\circ} \mathrm{C}$. GFP labels cells descended from the sal-LHG domain. (e) sal-LHG lexO-rpr L-trace Gal $80^{\text {ts }}$ disc after $1 \mathrm{~d}$ at $29^{\circ} \mathrm{C}$ and $3 \mathrm{~d}$ at $18^{\circ} \mathrm{C}$. GFP labels cells have experienced transient $r p r$ overexpression and their progeny. (f) A schematic of experiments in ( $\mathbf{g}-\mathbf{k})$. The blue line circles the sal domain. $(\mathbf{g}, \mathbf{h})$ lexO-rpr CasExpress $\mathrm{G}$-trace Gal80ts disc right after $1 \mathrm{~d}$ at $29^{\circ} \mathrm{C}(\mathbf{g})$ and after $3 \mathrm{~d}$ recovery at $18{ }^{\circ} \mathrm{C}(\mathbf{h})$. (i-k) sal-LHG lexO-rpr CasExpress G-trace Gal80ts disc right after $1 \mathrm{~d}$ at $29^{\circ} \mathrm{C}(\mathbf{i})$, after $3 \mathrm{~d}$ recovery at $18^{\circ} \mathrm{C}(\mathbf{j})$, and after $2 \mathrm{~d}$ recovery at $18^{\circ} \mathrm{C}(\mathbf{k})$. In (g-k), GFP marks cells that have survive executioner caspase activation. In (k), PH3 labels mitotic cells. White arrows point to several examples of mitotic CasExpress ${ }^{+}$cells. In all images, scale bar is $50 \mu \mathrm{m}$. 
was not overexpressed (Fig. 2g), we found increased CasExpress ${ }^{+}$ $\left(\mathrm{GFP}^{+}\right)$cells in the recovered epithelium after one day of $r p r$ overexpression at $29^{\circ} \mathrm{C}$ (Fig. 2i). After 3 days regeneration at $18^{\circ} \mathrm{C}$, few CasExpress ${ }^{+}$cells were present in the control without rpr overexpression (Fig. 2h), compared to the regenerated epithelium (Fig. 2j). The CasExpress ${ }^{+}$cells in the regenerated epithelium were at the same location (middle of the pouch) and abundance as those that survived transient $r p r$ overexpression (compare Fig. $2 \mathrm{j}$ to $\mathrm{e}$ ), indicating that most, if not all, rproverexpressing cells experienced executioner caspase activation. While many cells died, some survived and remained in the regenerated discs. After 2 days of regeneration, phospho-histone H3 (PH3) staining showed actively proliferating CasExpress ${ }^{+}$ cells (Fig. 2k-k"').

Developmental CasExpress activation depends on apoptosisinducing signals. We previously showed that $\sim 1 \%$ of cells in a growing disc activate CasExpress during any given 24-h period of normal disc development (Fig. 1d, m) ${ }^{11}$. Activation of CasExpress during normal development is dependent on the upstream caspase Dronc (Supplementary Figure S1g) ${ }^{11}$, and is thus distinct from the more recently described phenomenon of "basal" caspase activation in discs, which is Dronc-independent ${ }^{19}$. To gain further insight into the cellular and molecular mechanisms driving CasExpress activation, we set out to knockdown or overexpress genes and assess their effects on CasExpress. However, the original CasExpress employs Gal4/UAS and FLP/FRT, precluding Gal4/UAS-mediated RNAi or overexpression. To circumvent this limitation, we redesigned the CasExpress sensor. We replaced Gal4 with LexA and G-trace with L-trace, which is the LexAresponsive version of G-trace (Fig. 3a). Similar to the Gal4/UAS version, the LexA construct (henceforth referred to as L-CasExpress) showed a large fraction of $\mathrm{GFP}^{+}\left(\right.$CasExpress $\left.^{+}\right)$cells in third larval instar wing discs (Fig. 3b, b'). Although $\sim 50 \%$ of disc cells are $\mathrm{GFP}^{+}$by this stage, it is important to note that this reflects the sum of all cells that activate the sensor at any point in development and all of their progeny. Within any given $24 \mathrm{~h}$ period, only $\sim 0.9 \%$ cells activate CasExpress (Fig. $1 \mathrm{~d}, \mathrm{~m}$ ).

To test L-CasExpress activation for Dronc dependence and establish a quantitative RNAi screening assay, we used engrailedGal4 (en-Gal4) to express UAS-dronc RNAi specifically in the posterior compartment of the wing disc. The anterior compartment thus serves as an internal control. Compared to the white (w) RNAi control (Fig. 3c, c'), knocking down dronc blocked L-CasExpress activation (Fig. 3d, d') indicating that L-CasExpress, like the original CasExpress, is Dronc-dependent. To quantify the effect of dronc RNAi (or any UAS transgene) on LCasExpress activation, we calculated the ratio of the percentage of cells that were $\mathrm{GFP}^{+}$in the posterior compartment to that in the anterior compartment (CasExpress P/A) (Fig. 3e, j).

Using this assay, we found overexpression of $\mathrm{P} 35$, a baculovirus protein that specifically binds and inhibits the executioner caspases, virtually abolished L-CasExpress activation (Supplementary Figure S2a, b). We further tested which of the two Drosophila executioner caspases, Drice and Dcp-1, is required for CasExpress activation. We found that drice $R N A i$ reduced CasExpress activation (Fig. 3f, $\mathrm{f}$ and $\mathrm{j}$ ), whereas loss of $d c p-1$ had no measurable effect (Supplementary Figure S2c-e). This is notable because apoptosis induction in wing discs requires Drice but not Dcp-1 $1^{20,21}$, whereas basal caspase activation depends on both $^{19}$. Thus, CasExpress activation, like apoptotic induction ${ }^{20}$, reflects Drice activation during wing disc development.

Expression of $m i R G H$, a microRNA that targets three proapoptotic genes $r p r$, hid, and grim $^{22,23}$ also reduced CasExpress activation (Fig. 3g, g, j). Animals homozygous for rpr and grim, and heterozygous for hid, exhibited a nearly 20 -fold reduction in $\mathrm{GFP}^{+}$ imaginal disc cells (from 54 to 3\%, Supplementary Figure S2f-h). Therefore, developmental CasExpress activation, like apoptosisbut unlike basal caspase activity ${ }^{19}$-depends on Rpr, Hid, and Grim, Dronc, and Drice, but not Dcp-1. Although some cell types, such as sperm, require the upstream apoptotic cascade and caspase activation for normal differentiation 24,25 , the patterns of $\mathrm{L}$ CasExpress and such non-apoptotic caspase activation are different: Caspase is activated in every sperm cell at precisely the same stage of development ${ }^{25}$; whereas activation of CasExpress in the wing disc occurs in a subset of cells and is sporadic over time (ref. ${ }^{11}$ and this study). Together, the data, therefore, suggest that CasExpress ${ }^{+}$cells are cells that survive apoptotic executioner caspase activation during normal development.

These findings suggest that some cells sporadically experience endogenous, apoptosis-inducing stress during normal wing disc development, which is consistent with the fact that apoptotic cell death occurs in wing discs without any externally imposed stress $^{26}$. To test this hypothesis, we inhibited Jun N-terminal kinase (JNK), a well-established stress-activated kinase ${ }^{27}$. Overexpression of the JNK inhibitor puckered (puc) (Fig. 3h, h') or knockdown of the Drosophila JNK, called basket (bsk) (Fig. 3i, i') reduced L-CasExpress activation (Fig. 3j). Using a JNK activity reporter, TRE:DsRed ${ }^{28}$, we found that active JNK was present in the scutellum region but barely detectable in the pouch or hinge regions of normal wing discs (Fig. 3k, k'). Overexpression of $p 35$, which blocks the execution of apoptosis and thereby leads to the abnormal accumulation of stressed cells, caused an increase in the number of cells with activated JNK (Fig. 3l, l'), suggesting activated JNK normally eliminates stressed cells by apoptosis. These data together suggest that during development, transient apoptotic stresses activate JNK signaling, which in turn initiates executioner caspase activation. Cells that die are rapidly removed from the epithelium, while others survive as L-CasExpress ${ }^{+}$cells.

Identification of genes that regulate survival from stressinduced caspase activation. A key unanswered question is what molecular mechanisms rescue cells from stress-induced executioner caspase activation. To address this question, we crossed enGal4 and UAS-RNAi lines, together with the newly developed $L$ CasExpress sensor and L-trace, to screen for genes that enhanced or suppressed survival from stress-induced executioner caspase activation during development (Fig. 4a). We reasoned that genes associated with stress responses, growth, survival, and regeneration $^{29}$ would be good candidates.

We measured the percentage of cells with CasExpress activation in the RNAi-expressing compartment compared to the percentage in the control compartment (CasExpress $\mathrm{P} / \mathrm{A}$ ) (Fig. 3e). By taking the percentage, we normalized for general effects on growth and survival since these should equally affect $\mathrm{GFP}^{+}$and $\mathrm{GFP}^{-}$cells. The percentage of $\mathrm{GFP}^{+}$cells measures the frequency of survival from executioner caspase activation. A change in CasExpress activation could in principle result either from a change in the fraction of cells that activate executioner caspase and/or a change in the capacity to survive executioner caspase activation. To distinguish between these two possibilities, we used cDcpl staining to assess cell death. If a gene is required for cell survival from executioner caspase activation, we expect RNAi to cause an increase in the percentage of $\mathrm{cDcp}^{+}$dead cells (cells that die after executioner caspase activation) at the expense of CasExpress ${ }^{+}$cells (cells that live after executioner caspase activation). By contrast, if knocking down a gene affects apoptosis initiation and/or caspase activity but not the capacity to survive executioner caspase activation, we expect the percentages of CasExpress $^{+}$cells and of $\mathrm{cDcp}^{+}{ }^{+}$dead cells to change in the same 


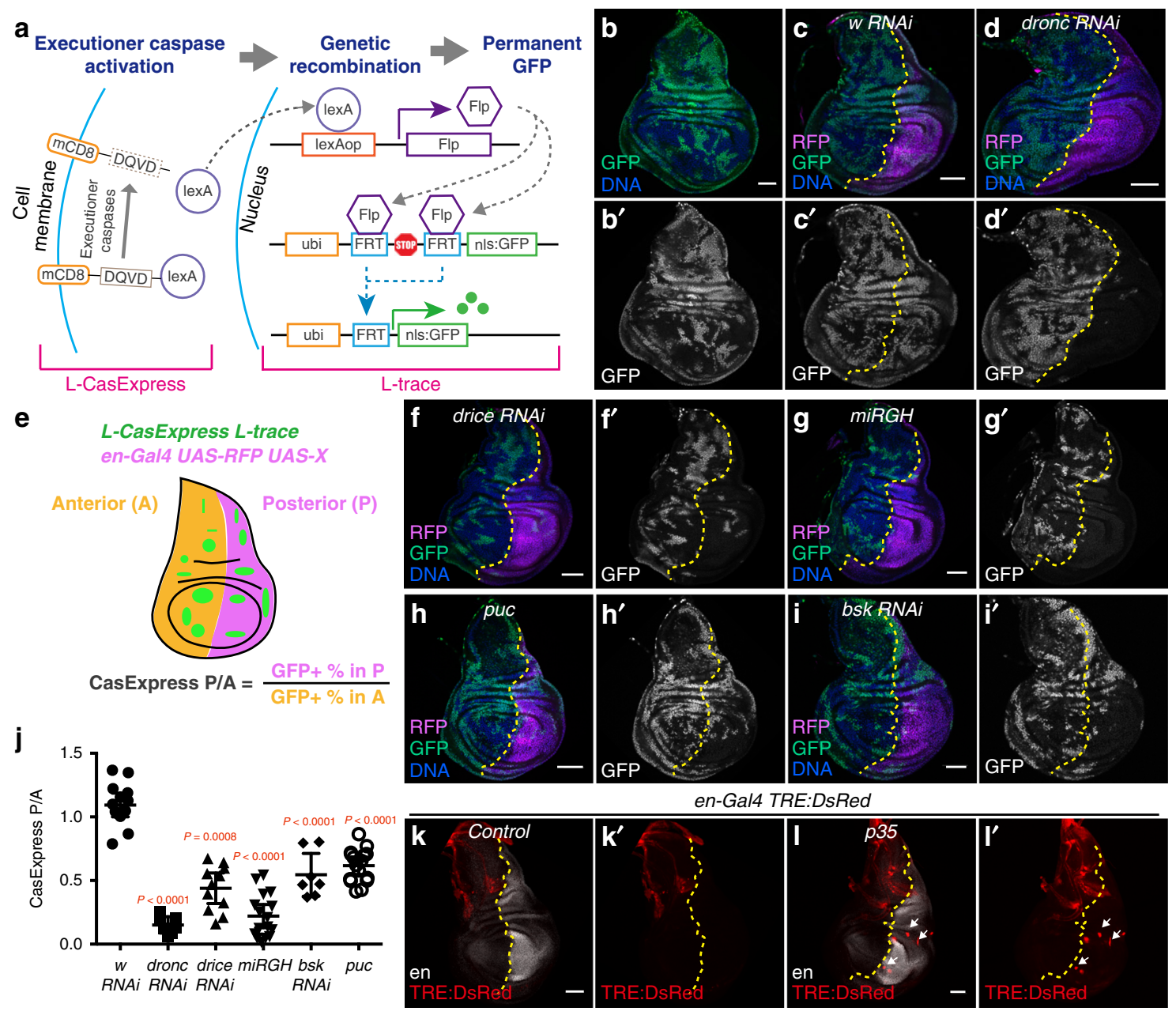

Fig. 3 Developmental CasExpress activation depends on apoptosis-inducing signals. (a) A schematic of L-CasExpress and L-trace. (b) L-CasExpress; Ltrace wing disc. GFP labels cells that survive executioner caspase activation. (c, d) Discs expressing enGal4, UAS-RFP, L-CasExpress, L-trace, and UAS-W RNAi (c) or UAS-dronc RNAi (d). (e) A schematic of the method for quantifying the effect of a genetic manipulation on survival from executioner caspase activation. A transgene $(X)$ is expressed in the posterior compartment of the wing disc under en-Gal4, and survival from executioner caspase activation in the whole disc is monitored by L-CasExpress. The effect of expression of $X$ on CasExpress activation is measured by the ratio of the percentage of GFP+ cells in the posterior compartment to that in the anterior compartment. (f-i) The effect of inhibiting apoptosis or JNK pathway on cell survival. Wing discs shown in the images expressed L-CasExpress, L-trace, en-Gal4, UAS-RFP, and UAS-drice RNAi (f), UAS-miRGH (g), UAS-puc (h), or UAS-bsk RNAi (i). (j) Quantification of CasExpress P/A ratio of the listed genotypes. $n=14$ (w RNAi), 7 (dronc RNAi), 11 (drice RNAi), 16 (miRGH), 7 (bsk RNAi), 16 (puc). $\mathrm{n}$ is the number of biological independent samples used for quantification. The data are presented as mean values $\pm 95 \%$ confidence interval. Statistical significance was determined after the logarithm transformation using one-way ANOVA. The Tukey test was used to derive adjusted $P$-value for multiple comparisons. The $\mathrm{P}$ values compared to $w$ RNAi are shown in the plot. Source data are provided as a Source Data file. (k, I) More cells with JNK activity were kept in the epithelium when execution of apoptosis was blocked (white arrows in I). Trachea attached to the disc are also DsRed ${ }^{+}$and not affected by p35 expression. In all images, RFP marks UAS transgene expressing region. The yellow dotted lines mark the boundary between the anterior compartment and the posterior compartment. Scale bar is $50 \mu \mathrm{m}$.

direction (Fig. 4a). However, due to the very low level of $\mathrm{cDcp}^{+}$ cells in control tissues, it is difficult to detect a significant reduction of $\mathrm{cDcpl}^{+}$cells. Thus, in discs with decreased CasExpress activation, if the percentage of $\mathrm{cDcp}^{+}$cells did not increase, we considered that the knockdown more likely affected caspase activation.

We screened 102 RNAi lines targeting 84 different genes which, when combined with en-Gal4, survived to late third instar (Supplementary data set 1). RNAi against the $w$ gene served as a negative control (Fig. 4b, b'). We found 13 genes that, when knocked down, increased both the percentage of CasExpress ${ }^{+}$ cells and the percentage of $\mathrm{cDcp}^{+}{ }^{+}$dead cells, indicating that knocking down these genes led to executioner caspase activation in more cells (Fig. 4a, Supplementary data set 1). (Note that any given cell is typically either CasExpress + and alive, or cDcp1+ and dead). Some known, general pro-survival and proliferation genes like thickveins (tkv), STAT92E, jub, Notch (n), appeared in this category (Supplementary Figure S3a-c, Supplementary data set 1). We found knockdown of any of 7 genes reduced the percentage of CasExpress ${ }^{+}$cells without elevating the percentage of $\mathrm{cDcp}^{+}$cells (Supplementary data set 1 ), suggesting knocking down these genes reduced executioner caspase activation frequency within the compartment. One gene in this category is expanded (ex), a known tumor suppressor. Knocking down ex abolished CasExpress ${ }^{+}$cells but did not induce extra $\mathrm{cDcp}^{+}$ cells (Figure S3d-f), consistent with the known effects of ex on expression of apoptosis inhibitors like Diap1 and bantam ${ }^{30}$. Interestingly, we identified 3 genes, akt1, bip2, and CG8108 for which RNAi resulted in a reduced percentage of CasExpress ${ }^{+}$ cells (Figs. 4c-d, 5a-c, Supplementary Figure S3g-h) and 
a

L-CasExpress L-trace

en-Gal4 UAS-RFP UAS-X

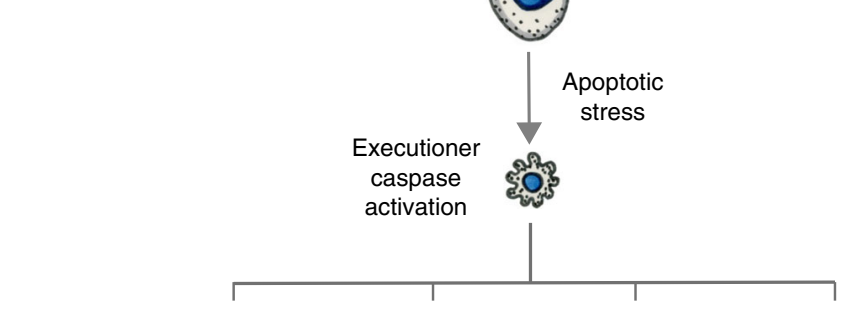

Survival
(CasExpress P/A) $\uparrow$

Death (cDcp1/RFP)

\begin{tabular}{c}
13 genes 7 genes \\
\hline $\begin{array}{c}\text { Regulators of survival before } \\
\text { executioner caspase activation }\end{array}$
\end{tabular}
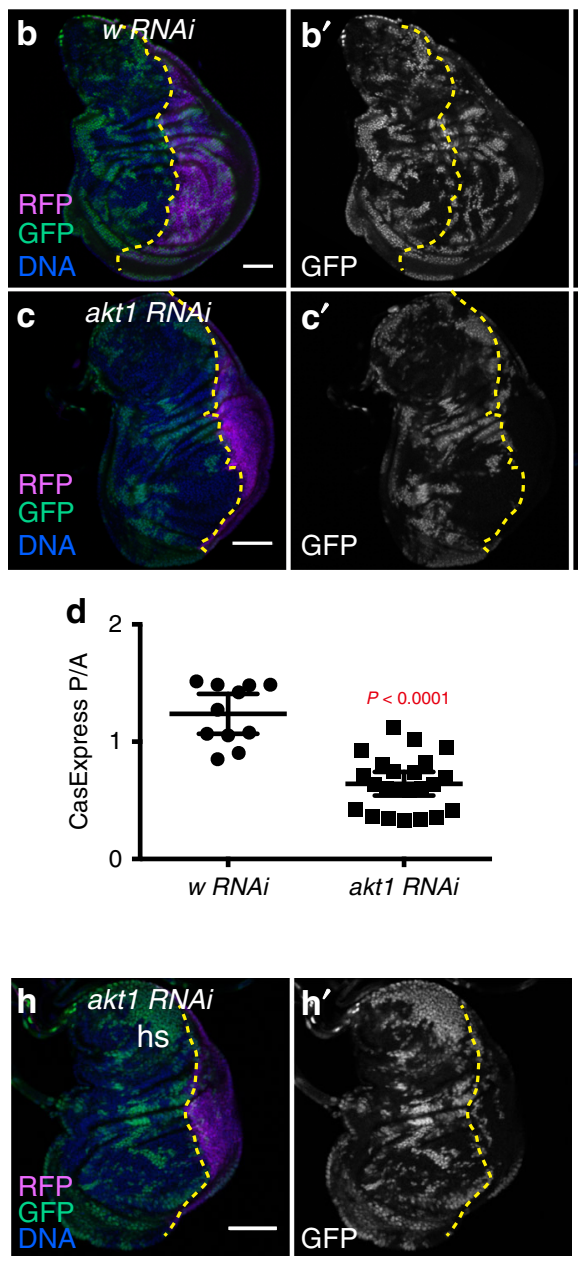
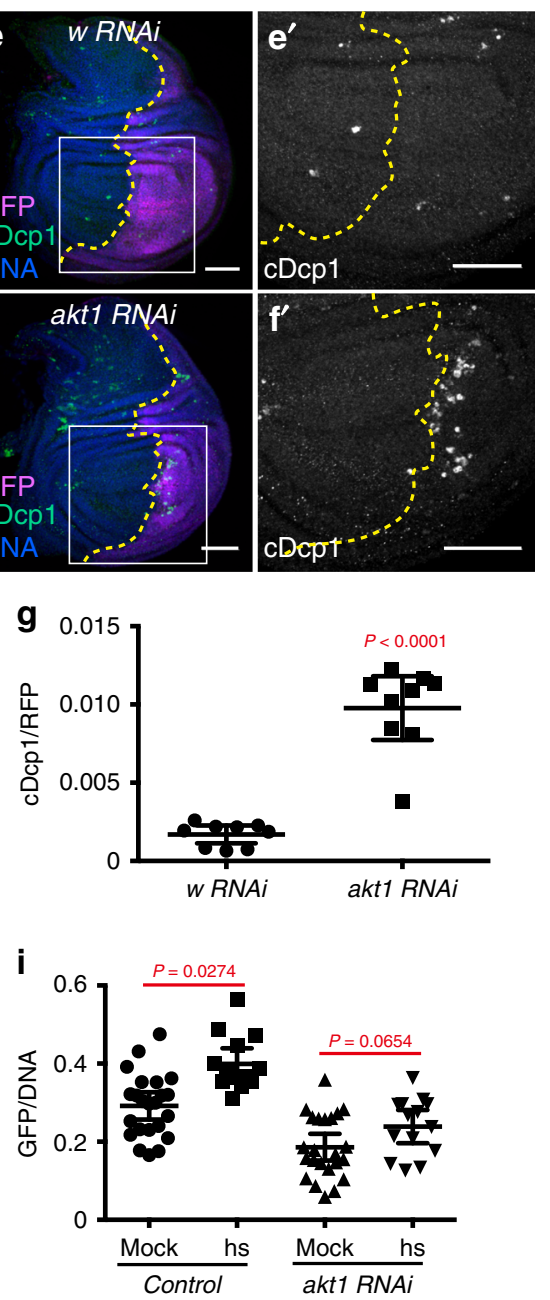

survival following an imposed apoptosis-inducing stress, we heatshocked enGal4; UAS-akt1RNAi larvae and evaluated the effect on L-CasExpress activation one day later. In this genotype, developmental L-CasExpress activation is not blocked before stress, so even unstressed (mock-treated) animals exhibit L-CasExpress activity. Nevertheless, heat shock-induced a significant increase in L-CasExpress activation in the anterior
Akt1 is required for survival from stress-induced executioner caspase activation. To test directly whether Akt1 is required for increased percentage of $\mathrm{CDcpl}^{+}$dead cells (Figs. $4 \mathrm{f}, \mathrm{g}, 5 \mathrm{~d}-\mathrm{f}$,
Supplementary Figure $\mathrm{S} 3 \mathrm{i}$ ), indicating these genes are required for survival from executioner caspase activation. 
Fig. 4 Identification of Akt1 as a promoter for survival from execution caspase activation through RNAi screening. (a) Classification and interpretations of RNAi effects on caspase activation followed by death (quantified by cDcp1 ${ }^{+}$area over RFP ${ }^{+}$area, cDcp1/RFP) or survival (quantified by CasExpress percentage in posterior compartment over that in anterior compartment, CasExpress P/A). n.c.: no change. (b-d) Knocking down akt1 for 3d suppressed CasExpress activation (GFP). The discs in (b) and (c) expressed enGal4, UAS-RFP, L-CasExpress, L-trace, Gal80ts and UAS-w RNAi (b) or UAS-akt1 RNAi (c). For the quantification in (d), $n=11$ (w RNAi), 23 (akt1 RNAi). (e-g) Knocking down akt1 for $3 \mathrm{~d}$ increased cell death (cDcp $1^{+}$). The discs in (e) and (f)

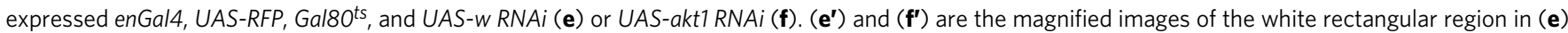
and (f). For the quantification in ( $(\mathbf{g}), n=9$ for both genotypes. (h) Heat shock cannot induce CasExpress activation in akt7-knocked-down compartment. (i) Quantification of the percentage of GFP+ cells in the anterior compartment (control) and in the akt1 RNAi compartment in mock-treated or heat-shocked discs. $n=23$ (mock), 14 (hs). In all images, RFP marks UAS transgene expressing region. The yellow dotted lines mark the boundary between the anterior compartment and the posterior compartment. Scale bar is $50 \mu \mathrm{m}$. In all dot plots, $\mathrm{n}$ is the number of biological independent samples used for quantification. The data are presented as mean values $\pm 95 \%$ confidence interval. ns: no statistical significance. Statistical significance was determined after logarithm transformation using unpaired two-tailed $t$-test. Source data are provided as a Source Data file.

compartment of the wing discs, where akt1 RNAi was not expressed (Fig. 4h, h', i). In contrast, no significant heat-shockinduced increase in L-CasExpress activation was detected in the akt1 RNAi-expressing compartments (Fig. 4h, h', i).

CG8108, a Drosophila homolog of CIZ1, is essential for survival from executioner caspase activation. Of the 102 RNAi lines we tested for effects on CasExpress activation, two (BL\#36665 and BL\#28329) were supposed to target the gene chico. When expressed with en-Gal4, both reduced CasExpress activation (Fig. 5a, a', c, Supplementary Figure S4a, b). However, expression of BL\#28329 dramatically increased the number of $\mathrm{cDcp} 1^{+}$cells (Fig. 5d, d', f) whereas BL\#36665 did not (Supplementary Figure S4a"). We thought that BL\#28329 might provide a stronger knockdown than BL\#36665, so we used qPCR to compare their effects on chico mRNA levels. Surprisingly, BL\#36665 reduced chico mRNA level to one-fourth, whereas BL\#28329 had no measurable effect (Figure S4c), indicating BL\#28329 did not target chico.

To identify the likely target of this RNAi line, we sequenced the hairpin and found that BL\#28329 targeted CG8108, rather than chico, indicating that it was mislabeled in the stock center. We confirmed with qPCR that expression of BL\#28329 reduced the mRNA level of CG8108 by half (Figure S4d).

CG8108 is an uncharacterized gene encoding a protein homologous to human CDKN1A-interacting zinc finger protein 1 (CIZ1). A second RNAi line against dCIZ1 (CG8108) (BL\#27562) caused similar phenotypes as BL\#28329, including reduced CasExpress activation (Fig. 5b, b', c) and increased $\mathrm{cDcp}^{+}$cells (Fig. 5e, e', f). These phenotypes were rescued by overexpression of dCIZ1 (Fig. 5g-j). Furthermore, dCIZ1 knockdown prevented heat-shock- or radiation-induced L-CasExpress activation (Fig. 5k-m). These data together suggest dCIZ1 is essential for survival from executioner caspase activation.

Overexpression of dCIZ1 was not sufficient to protect against $r p r$-induced apoptotic cell death (Supplementary Figure S5a-c). There was no cleaved form of dCIZ1 specific to apoptotic cells (Supplementary Figure S5d). Nor was there a detectable physical interaction between dCIZ1 and Drice or cleaved Drice (Supplementary Figure S5e), suggesting dCIZ1 may not promote survival through direct interaction with Drice.

dCIZ1 required for survival from executioner caspase activation in tissues with activated PI3K/Akt signaling. The phosphinositide-3-kinase (PI3K) pathway (Supplementary Figure $\mathrm{S6a}$ ) is associated with cell proliferation and survival in many contexts $^{31}$. RNAi screening revealed potent effects-some expected and others not-of multiple components of PI3K pathway on cell death and on CasExpress activation (Supplementary Figure S6b-g, Supplementary data set 1). For example, the phosphatase and tensin homolog (PTEN) is a negative regulator of PI3K signaling and an established tumor suppressor ${ }^{31}$. RNAi against pten resulted, unsurprisingly, in compartment overgrowth (Fig. 6a, a', f). However, pten RNA $i$ also resulted in increased apoptotic cell death detected with anti-cDcpl staining (Fig. 6a, a'), suggesting that tissue overgrowth due to hyperactive PI3K signaling might cause stress and induce apoptosis. Increased CasExpress activation in the pten RNAi-expressing compartment (Fig. 6b, c) shows not only increased death but also increased survival following apoptotic caspase activation.

The pattern of increased $\mathrm{cDcp} 1$ and CasExpress indicates that pten loss increased apoptotic induction of caspase activity (Fig. 4a). To test this idea, we inhibited $\mathrm{rpr} / \mathrm{hid} / \mathrm{grim}$ expression in cells expressing pten RNAi. Overexpressing $m i R G H$ in the wing disc compartment expressing pten RNAi reduced CasExpress activation by two-thirds (Fig. 6d, e) and virtually eliminated $\mathrm{cDcp}^{+}$dead cells (Fig. 6d"). Thus, the executioner caspase activation and cell death within the pten RNAi compartment required pro-apoptotic genes. The observation that $m i R G H$ expression in the pten RNAi domain further increased the compartment size by $15 \%$ (Fig. 6f), suggests that apoptosis restrained tissue overgrowth to some extent.

To test which effects of pten RNAi were consequences of activation of PI3K pathway, we manipulated PI3K signaling in the pten RNAi compartment. Knocking down the catalytic subunit of the PI3K complex, pi3k92e, together with pten RNAi suppressed CasExpress activation, $\mathrm{cDcp}^{+}$dead cells (Fig. $6 \mathrm{~g}, \mathrm{~h}$ ), and overgrowth (Fig. 6i). Furthermore, overexpression of pi3k92e elevated both cell death (Fig. 6j) and CasExpress activation (Fig. 6k, 1). Similar to pten RNAi, pi3k92e-induced CasExpress activation and cell death was inhibited by overexpression of miRGH (Fig. $6 \mathrm{~m}, \mathrm{n}$ ). Constitutively activated akt1 (myr:akt1) caused similar effects (Fig. 6o-s). These data suggest that activation of PI3K-Akt1 signaling promotes tissue overgrowth, which results in apoptotic stress in the overgrown compartment, possibly due to overgrowthinduced cell crowding 32 and/or competition for nutrients or survival factors ${ }^{33,34}$. However, activated Akt1 also promotes survival from executioner caspase activation, which contributes to tissue overgrowth (Fig. 7i). These results underscore the multifaceted effects of PI3K/Akt signaling on multicellular tissues.

The discovery of $\mathrm{dCIZ1}$ as an important regulator of cell survival from caspase activation during normal development raised the question as to whether survival from executioner caspase activation generally requires dCIZ1. We found that CasExpress activation in pi3k92e-overexpressing discs (Fig. 7a-a") was eliminated by dCIZ1 knockdown (b-b" and f), indicating an essential role of dCIZ1 in promoting survival in the context of activated PI3K. Next, we tested the effect of $d C I Z 1$ RNA $i$ on myr: akt1-induced CasExpress activation. Knocking down dCIZ1 significantly suppressed myr:akt1-induced CasExpress activation (Fig. 7c-e, g). Because both UAS-myr:akt1 and the two 

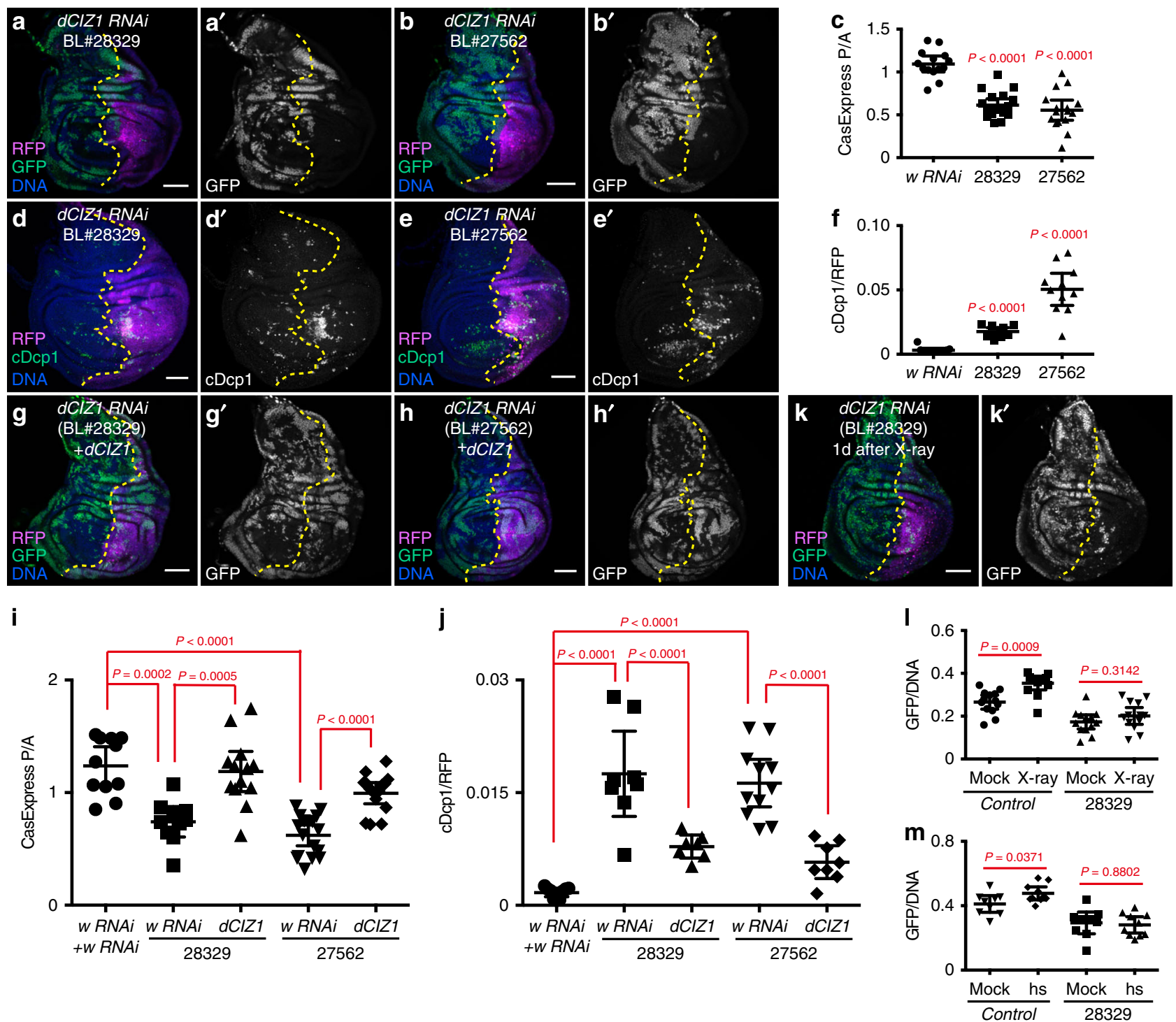

Fig. 5 dCIZ1 is essential for survival from executioner caspase activation. (a, b) Expression of two different RNAi against $d C I Z 1$ reduced CasExpress activation (GFP). (c) Quantification of CasExpress activation in discs expressing enGal4, UAS-RFP, L-CasExpress, L-trace, and $w$ RNAi ( $n=14)$ or BL\#28329 $(n=18)$ or $B L \# 27562(n=16)$. (d, e) Expression of two different RNAi against $d C I Z 1$ increased cell death $\left(c D c p 1^{+}\right)$. (f) Quantification of $c D c p 1^{+}$area in compartments expressing w RNAi $(n=11)$ or $B L \# 28329(n=10)$ or $B L \# 27562(n=11)$ (marked by RFP). (g-i) Overexpression of $d C I Z 1$ in $d C l Z 1$ RNAi compartment restored CasExpress activation (GFP). $n=11(w R N A i+w R N A i), 10(28329+w R N A i), 13(28329+d C I Z 1), 17(27562+w R N A i), 15(27562$ $+d C I Z 1)$. (j) Overexpression of $d C I Z 1$ suppressed cell death induced by $d C I Z 1 R N A i . n=9$ ( $W R N A i+w R N A i), 8(28329+w R N A i), 7(28329+d C I Z 1), 11$ $(27562+w R N A i), 8(27562+d C I Z 1)$. (k) radiation cannot induce CasExpress activation (GFP) in dCIZ1 RNAi compartment. (I) Quantification of the percentage of $\mathrm{GFP}^{+}$cells in the anterior compartment (control) and in the $d C I Z 1$ RNAi (BL\#28329) compartment in mock-treated (mock) or radiated discs (X-ray). $n=13$ for both groups. ( $\mathbf{m}$ ) Heat shock cannot induce CasExpress activation (GFP) in dCIZ1 RNAi (BL\#28329) compartment. The plot shows quantification of the percentage of GFP+ cells in the anterior compartment (control) and in the $d C I Z 1$ RNAi compartment in mock-treated or heat-shocked discs. $n=9$ (mock), 10 (hs). In all images, RFP marks UAS transgene expressing region. The yellow dotted lines mark the boundary between the anterior compartment and the posterior compartment. Scale bar is $50 \mu \mathrm{m}$. In all plots, $\mathrm{n}$ is the number of biological independent samples used for quantification. The data are presented as mean values $\pm 95 \%$ confidence interval. Statistical significance was determined after logarithm transformation using unpaired two-tailed $t$-test $(\mathbf{I}, \mathbf{m})$ or one-way ANOVA $(\mathbf{c}, \mathbf{f}, \mathbf{i}, \mathbf{j})$. The Tukey test was used to derive the adjusted $P$-value for multiple comparisons. Source data are provided as a Source Data file.

Bloomington $d C I Z 1$ RNA $i$ are on the third chromosome, we used another dCIZ1 RNAi from Vienna Drosophila Resource Center, vdrc35344, in this experiment. Similar to BL\#28329 and $B L \# 27562$, expression of vdrc35344 increased the fraction of $\mathrm{cDcp}^{+}$dead cells (Supplementary Figure S7a). The increased cell death in vdrc35344-expressing discs was rescued by co-expressing $d C I Z 1$ (Supplementary Figure S7b), suggesting vdrc35344 targets on $d C I Z 1$.
It has been reported that knocking down CIZ1 in human prostate cancer cells reduced Akt expression ${ }^{35}$. However, neither the Akt level (Fig. 7h) nor the PI3K-induced Akt phosphorylation was affected in $d C I Z 1$ RNA $i$ discs (compare Fig. 7a", b"). These data together are consistent with a function for dCIZ1 downstream of or in parallel to Akt1 (Fig. 7i), although we cannot formally exclude the possibility that dCIZ1 may act upstream of Akt1. 

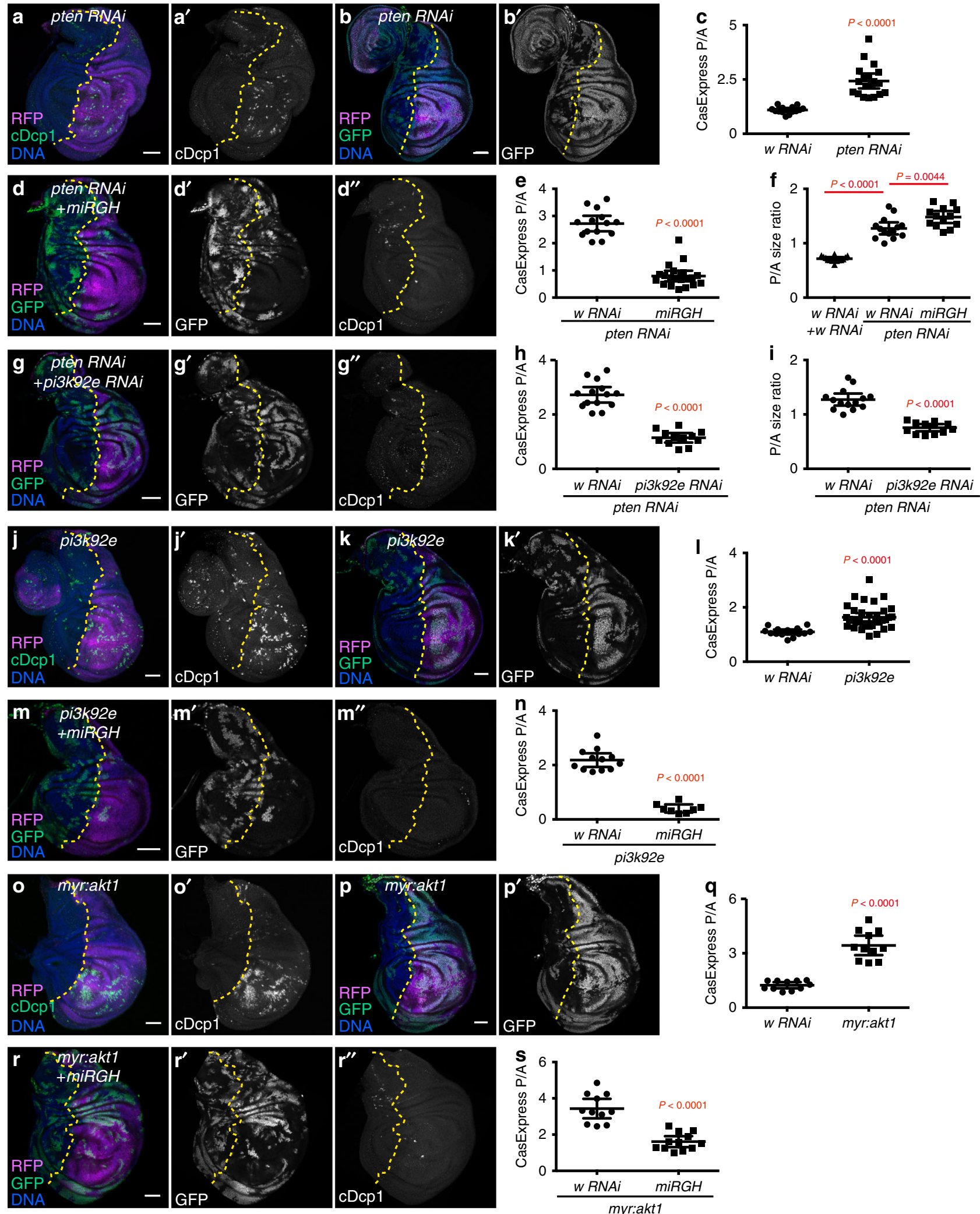

Akt1 and dCIZ1 promote survival of cells with oncogenic Ras $^{\mathrm{V} 12}$-induced executioner caspase activation. Hyperactivation of PI3K-Akt1 signaling occurs in a large variety of human cancers. The human PIK3CA gene, which encodes the catalytic subunit of the PI3K complex, is the second most frequently mutated oncogene, and PTEN is one of the most frequently mutated tumor suppressor genes ${ }^{31}$. The discovery of induced

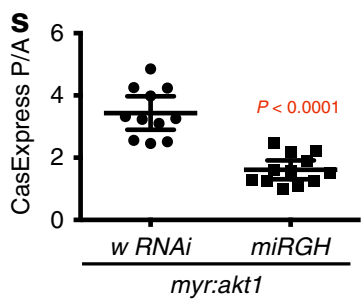

apoptotic stress and survival from executioner caspase activation in tissues with activated PI3K-Akt signals led us to test whether expression of another common oncogenic mutation, Ras ${ }^{\mathrm{V} 12}$, would also induce autonomous apoptotic stress and subsequent survival from executioner caspase activation. Expression of ras $^{V 12}$ with en-Gal4 was lethal, so we used Gal80 ts to limit the expression to three days. Expression of oncogenic ras $^{V 12}$ in the posterior 
Fig. 6 Activation of PI3K-Akt1 signaling induces autonomous apoptotic stress and promotes survival from executioner caspase activation. (a-c) Knocking down pten induced cell death $\left(\mathrm{cDcp}^{+}\right)$(a) and increased CasExpress activation (GFP) (b, c). $n=14$ (w RNAi), 19 (pten RNAi). (d, e) Overexpression of miRGH in the pten RNAi compartment strongly reduced CasExpress activation and eliminated cell death. $n=14$ (pten RNAi+w RNAi), 20 (pten $R N A i+m i R G H)$. (f) Knocking down pten caused overgrowth of posterior compartment (quantified by the ratio of the posterior compartment size over the anterior compartment size, P/A size ratio), which was further increased by co-overexpression of miRGH. $n=14$ ( $w R N A i+w R N A i), n=14$ (pten RNAi $+w R N A i), 13$ (pten RNAi + miRGH). (g-h) Knocking down pi3k92e suppressed the elevated CasExpress activation and cell death caused by pten RNAi. $n=$ 14 (pten $R N A i+w R N A i$ ), 12 (pten RNAi + pi3k92e RNAi). (i) Knocking down pi3k92e suppressed overgrowth of the pten RNAi compartment. $n=14$ (pten $R N A i+w R N A i), 12$ (pten $R N A i+$ pi3k92e RNAi). (j-I) Overexpression of pi3k92e increased cell death (cDcp ${ }^{+}$) (j) and CasExpress activation (GFP) (k, I). $n=14$ ( $w R N A i), 34$ (pi3k92e). ( $\mathbf{m}, \mathbf{n}$ ) Overexpression of miRGH blocked both CasExpress activation and cell death. $n=12$ (pi3k92e $+w R N A i), 8$ $\left(\right.$ pi3k92e + miRGH). (o-q) Overexpression of myr:akt1 for 3d increased cell death (cDcp $\left.1^{+}\right)(\mathbf{o})$ and CasExpress activation (GFP) (p-q). $n=11(w R N A i)$, 11 (myr:akt1). (r, s) Overexpression of miRGH suppressed myr:akt1-induced elevation in CasExpress activation and cell death. $n=11$ (myr:akt1 + w RNAi), 12 (myr:akt1 + miRGH). Discs used in (o-s) contained Gal80ts to control the expression timing of myr:akt1. In all images, RFP marks UAS transgene expressing region. The yellow dotted lines mark the boundary between the anterior compartment and the posterior compartment. Scale bar is $50 \mu \mathrm{m}$. In all plots, $\mathrm{n}$ is the number of biological independent samples used for quantification. The data are presented as mean values $+ \pm 95 \%$ confidence interval. Statistical significance was determined after logarithm transformation using unpaired two-tailed t-test (all plots other than $\mathbf{f}$ ) or one-way ANOVA (f). The Tukey test was used to derive adjusted P-value for multiple comparisons. Source data are provided as a Source Data file.
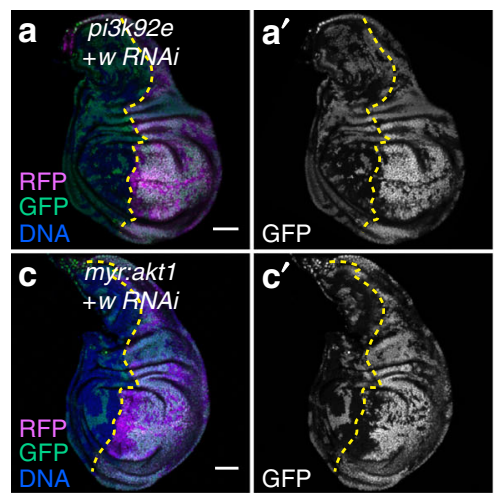

f

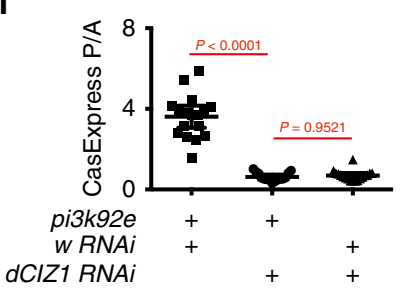

(BL\#28329)
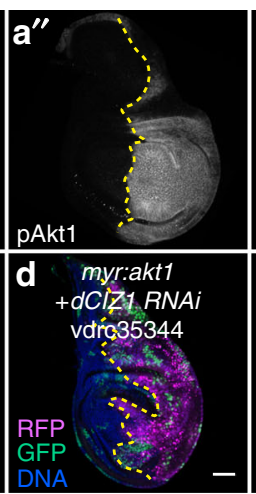

g

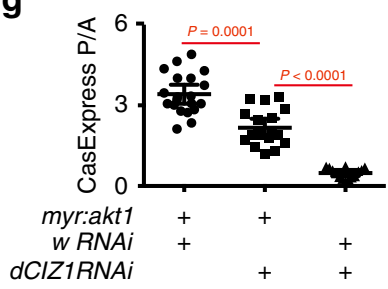

(vdrc35344)
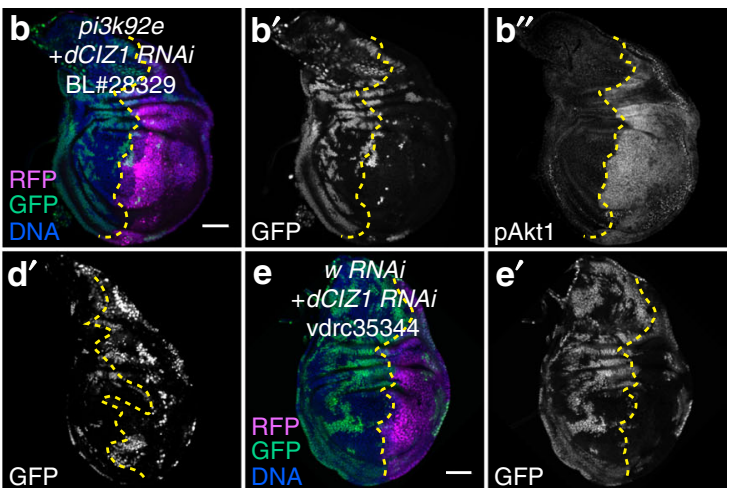

h
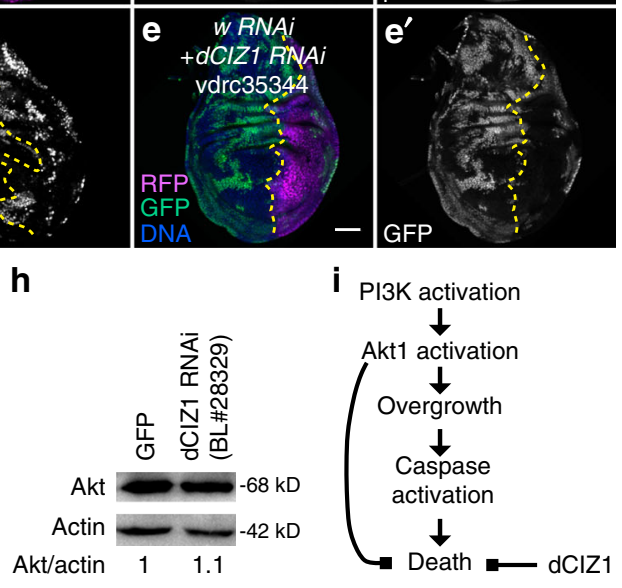

i PI3K activation

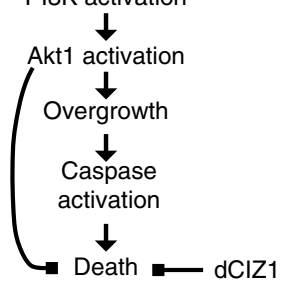

Fig. 7 dClZ1 promotes survival from executioner caspase activation in PI3K- or Akt1-activated tissues. (a, b) Knocking down $d C l Z 1$ suppressed CasExpress activation but not Akt1 activation in the compartment expressing pi3k92e. dCIZ1 RNAi (BL\#28329) was used in (b). (c-e) Knocking down dCIZ1 suppressed myr:akt1-induced CasExpress activation. dCIZ1 RNAi (vdrc35344) was used in (c-e), and Gal80 ts was used to limit expression of the transgenes to 3 days. (f) Quantification of CasExpress P/A in discs expressing L-CasExpress, L-trace, en-Gal4, UAS-RFP, together with UAS-pi3k92e and UAS-w RNAi $(n=17)$, or UAS-pi3k92e and UAS-dCIZ1 RNAi (BL\#28329) ( $n=15)$, or UAS-dCIZ1 RNAi (BL\#28329) and UAS-w RNAi $(n=18)$. (g) Quantification of CasExpress P/A in discs expressing L-CasExpress, L-trace, en-Gal4, UAS-RFP, tubGal80ts together with UAS-myr:akt1 and UAS-w RNAi ( $n=20)$, or UAS-myr: akt1 and UAS-dCIZ1 RNAi (vdrc35344) ( $n=18$ ), or UAS-dCIZ1 RNAi (vdrc35344) and UAS-w RNAi $(n=14)$. (h) Expression of $d C I Z 1$ RNAi (BL\#28329) under en-Gal4 did not affect $\mathrm{dClZ1}$ protein level. The numbers below the blots are the relative ratio between the intensity of Akt band and that of actin band. (i) The schematic of the role of Akt1 and dCIZ1 in promoting survival from executioner caspase activation in tissues with activated PI3K-Akt1 signaling. In all images, RFP marks UAS transgene expressing region. The yellow dotted lines mark the boundary between the anterior compartment and the posterior compartment. Scale bar is $50 \mu \mathrm{m}$. In all plots, $\mathrm{n}$ is the number of biological independent samples used for quantification. The data are presented as mean values $\pm 95 \%$ confidence interval. Statistical significance was determined after logarithm transformation using one-way ANOVA. The Tukey test was used to derive adjusted P-value for multiple comparisons. Source data are provided as a Source Data file.

compartment led to a more than two-fold increase in the fraction of cells with CasExpress activation (Fig. 8a, b). Strikingly, few if any $\mathrm{cDcp}^{+}$cells were detectable (Fig. 8c), indicating that most cells that activated executioner caspases survived. Co-expressing $m i R G H$ to inhibit apoptosis induction in the ras ${ }^{V 12}$-expressing compartment suppressed the elevated CasExpress (Fig. 8b, d). We conclude that ras $^{V 12}$-induced CasExpress activation requires apoptosis induction.
Mild activation of executioner caspases in $s r i b^{-}$ras $^{V 12}$ clones in eye discs can promote neoplastic growth ${ }^{36}$. However, in ras $^{V 12}$ expressing disc cells, overexpression of miRGH caused a small increase rather than reduction in the compartment size (Fig. 8e). Therefore, apoptosis restrains the overgrowth of tissues with oncogenic mutations including both ras $^{V 12}$ and, as shown above, PI3K. Counteracting this growth-inhibiting apoptosis, Ras V12 also promotes survival from executioner caspase activation, which 

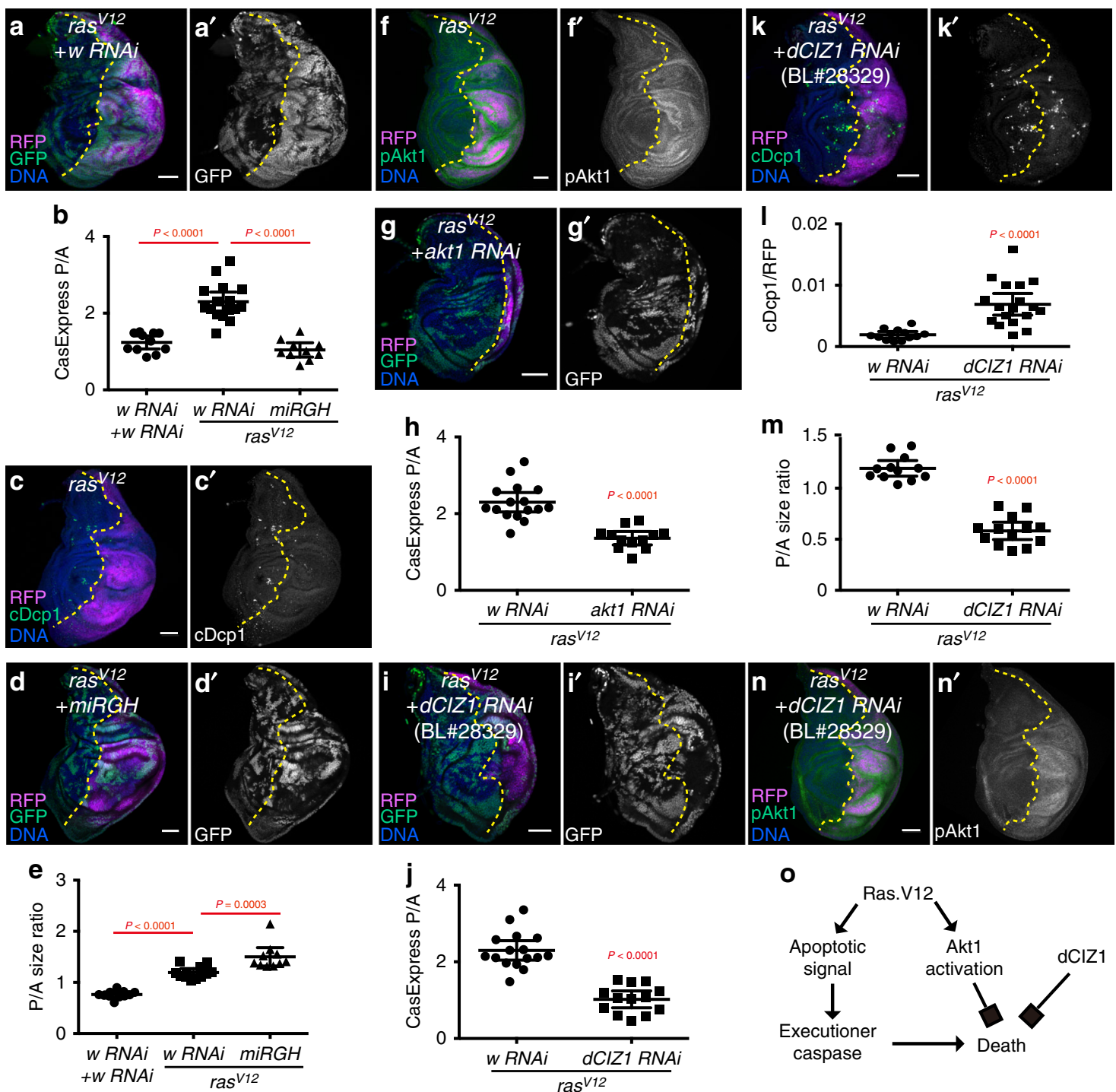

Fig. 8 Akt1 and dCIZ1 promote survival from apoptotic executioner caspase activation in tissues with oncogenic Ras $\mathbf{V 1 2}$. (a) Expression of ras $V 12$ induced CasExpress activation (GFP). (b) Quantification of CasExpress activation in discs expressing $w R N A i+w R N A i(n=11)$, ras $V 12+w R N A i(n=16)$, ras ${ }^{12}+\operatorname{miRGH}(n=10)$ under en-Gal4. (c) Expression of ras V12 induced little cell death. (d) miRGH inhibited CasExpress activation in ras $V 12$-expressing compartment. (e) Expression of ras $V 12$ induced overgrowth of the compartment, which was further increased by overexpression of miRGH. $n=11$ ( $w$ RNA + w RNAi), 12 (ras V12 + w RNAi), 10 (ras ${ }^{V 12}+$ miRGH). (f) Expression of ras V12 activated Akt1. (g, h) Knocking down akt1 suppressed ras V12 -induced CasExpress activation. $n=16\left(\right.$ ras $^{V 12}+w$ RNAi), 12 (ras V12 + akt1 RNAi). (i, j) Knocking down dCIZ1 blocked CasExpress activation in ras $V 12$-expressing compartment. $n=16$ (ras V12 $+w R N A i), 13$ (ras V12 $+d C I Z 1$ RNAi). (k, I) Knocking down dCIZ1 induced cell death (cDcp1 $\left.{ }^{+}\right)$in ras ${ }^{V 12}$-expressing compartment. $n=13$ (ras V12 $+w$ RNAi), 18 (ras V12 $+d C I Z 1$ RNAi BL\#28329). (m) Knocking down $d C I Z 1$ suppressed ras V12 induced overgrowth. $n=12$ $\left(\right.$ ras $^{V 12}+$ w RNAi) 13 (ras V12 + dCIZ1 RNAi BL\#28329). (n) Expression of dCIZ1 RNAi (BL\#28329) did not affect ras ${ }^{V 12}$-induced Akt1 activation. (o) The schematic of the role of $\mathrm{Akt1}$ and $\mathrm{dCIZ1}$ in tissues with oncogenic Ras ${ }^{\mathrm{V} 12}$. Gal80ts was used in the experiments in this figure to control the timing of ras $\mathrm{V} 12$ expression. In all images, RFP marks UAS transgene expressing region. The yellow dotted lines mark the boundary between the anterior compartment and the posterior compartment. Scale bar is $50 \mu \mathrm{m}$. In all plots, $n$ is the number of biological independent samples used for quantification. The data are presented as mean values $\pm 95 \%$ confidence interval. Statistical significance was determined after logarithm transformation using unpaired two-tailed $t$-test $(\mathbf{h}, \mathbf{j}, \mathbf{I}, \mathbf{m})$ or one-way ANOVA $(\mathbf{b}, \mathbf{e})$. The Tukey test was used to derive adjusted $P$-value for multiple comparisons. Source data are provided as a Source Data file.

eventually contributes to tissue overgrowth. Promoting survival from caspase activation due to apoptotic stress represents a previously unknown function for activated Ras.

Consistent with a previous report that PI3K signaling is activated in $\mathrm{ras}^{\mathrm{V} 12}$-expressing cells ${ }^{37}$, we detected increased active Akt1 (phospho-Akt1) in the ras ${ }^{V 12}$-expressing compartment (Fig. 8f). Knocking down akt1 in the ras ${ }^{V 12}$-expressing compartment blocked the induced CasExpress activation (Fig. 8g-h), suggesting $\mathrm{Ras}^{\mathrm{V} 12}$ promotes survival from executioner caspase activation through Akt1. Knocking down dCIZ1 in ras ${ }^{V 12}$ overexpressing discs also suppressed CasExpress activation (Fig. 8i, j), induced apoptotic cell death (Fig. 8k, l), and suppressed Ras ${ }^{\mathrm{V} 12}$-induced tissue overgrowth (Fig. 8m). Compartments co-expressing $d C I Z 1$ RNAi and ras V12 showed high Akt1 activity (Fig. 8n), again indicating dCIZ1 may function downstream of or in parallel to Akt1.

Based on these data, we propose that during Ras ${ }^{\mathrm{V} 12}$-induced oncogenic transformation, competing death and survival signals 
are activated. Apoptosis is initiated and executioner caspases are activated, either as a homeostatic mechanism or as a consequence of nutrient-deprivation, hypoxia, or mechanical stress. In any case, dCIZ1 and activated Akt1 in the transformed cells promote survival of stressed cells, thereby contributing to oncogenic overgrowth (Fig. 80).

\section{Discussion}

Here we show that cells can survive executioner caspase activation induced by apoptotic signals during normal development, following imposed apoptosis-inducing stresses such as heat shock, radiation, and $r p r$ overexpression, and in response to oncogene activation. We show that cells can survive executioner caspase activation and contribute to tissue regeneration following severe imposed stresses. The wing disc is a highly regenerative tissue. It can regenerate even after half of the cells $\operatorname{die}^{38}$. A widelyacknowledged mechanism underlying wing disc regeneration is apoptosis-induced proliferation, in which apoptotic cells emit mitogenic signals to promote proliferation of surrounding healthy cells ${ }^{18,39-41}$. The initiator caspase Dronc is required for mitogenic signal production in apoptotic cells ${ }^{42}$. Apoptosis and executioner caspases also play indispensable roles in regeneration in other model organisms like planaria, hydra, and mice ${ }^{43-47}$. For example, loss of either caspase- 3 or -7 impairs skin wound repair and liver regeneration in mice ${ }^{44}$. In the classic apoptosis-induced regeneration model, cells initiate apoptosis, activate executioner caspases, and die; the regenerated epithelium originates from healthy cells that never activate executioner caspase. Here we report that even cells that activate executioner caspases can survive and participate in regeneration.

The fraction of the regenerated epithelium that originates from cells that survive apoptotic caspase activation varies depending on the conditions. In irradiated larvae, the majority of cells in the recovered disc are progeny of cells that have experienced executioner caspase activation (Fig. 1). In contrast, when discs regenerate after local and transient $r p r$ overexpression, fewer CasExpress $^{+}$cells contribute to recovery (Fig. 2). The difference could be due to different rates or levels of caspase activation ${ }^{20}$ and/or to competition from unstressed cells ${ }^{48,49}$. In the radiation experiments, all cells were stressed whereas $r p r$ overexpression was limited to a small fraction of the disc, so there was a large pool of unstressed cells nearby. These findings suggest that salvaging cells from executioner caspase activation may be particularly important for recovery from a transient apoptosisinducing stress that affects an entire organ or organism, such as systemic chemotherapy.

One hallmark of cancer is the capacity to evade cell death. An apparent paradox is that caspase activity is prevalent in gastric and breast cancers, melanoma, and lymphoma ${ }^{50-52}$, amongst others. In recent years, apoptosis and caspases have been shown to promote tumor growth, recurrence, and metastasis both autonomously and non-autonomously ${ }^{36,53-57}$. After radiation or chemotherapeutic drug treatment, caspase 3-dependent secretion of growth factors in apoptotic tumor cells can trigger repopulation of non-apoptotic tumor cells and angiogenesis ${ }^{54-56,58}$. Apoptotic lymphoma cells can modify the tumor microenvironment and activate tumor-associated macrophages to promote tumor progression ${ }^{57}$.

In addition to these paracrine effects on tumor progression, executioner caspase activity can also autonomously drive tumor growth and metastasis in Drosophila ${ }^{36}$ and mouse models ${ }^{53}$. In this study, we found that PI3K activation and oncogenic Ras V12 mutation, two of the most frequent molecular alterations in tumors, can promote cell survival from apoptotic executioner caspase activation. Our observation that inhibition of survival from executioner caspase activation in ras $^{\mathrm{V} 12}$-expressing tissues suppressed overgrowth demonstrates that recovery from apoptotic caspase activation represents a previously unrecognized mechanism by which Ras ${ }^{\mathrm{V} 12}$ promotes survival.

We identified Aktl as an essential driver for survival from executioner caspase activation. As a pro-survival protein, Akt is well known to inhibit apoptosis upstream of caspase activation and mitochondrial permeabilization ${ }^{59,60}$. Our work reveals the capacity of Akt1 to promote cell survival after executioner caspase activation in response to apoptotic stresses, consistent with one study that shows that Akt can inhibit apoptosis even after mitochondrial outer membrane permeabilization, at least in one cell type ${ }^{61}$. Khalil et al. ${ }^{62}$ found that exposure of multiple cell types and tissues to apoptosis-inducing radiation or chemicals results in stress-induced Akt activation that is dependent upon caspase 3 induction of apoptosis and necessary for tissue repair and animal survival. Though they did not show Akt was activated in the very same cells that activated caspase 3, since they did not have a CasExpress-like reporter, it seems likely that they detected Akt-mediated survival after executioner caspase activation. These, together with our data, suggest the regulation of survival from executioner caspase activation may be conserved from flies to mammals.

It is exciting then, that in addition to Ras and PI3K/Akt1, the fly screen revealed the importance of CG8108 (dCIZ1). Though there are no published functional studies on dCIZ1, it was identified in the interactome of stress-activated MAP kinases ${ }^{63}$. Remarkably, it also emerged in a PI3K interactome ${ }^{64}$. The human homolog of dCIZ1 has many attributes consistent with a determinant that promotes survival in the face of tissue damage. For example, CIZ1 binds and inactivates the senescence-promoting protein $\mathrm{p} 21^{\mathrm{Cip} 1 / \mathrm{Waf} 165}$, thus antagonizing DNA-damage-induced proliferation arrest. It has reported roles in DNA replication ${ }^{66}$, cell cycle progression 67 , transcription ${ }^{68-70}$, and epigenetic regulation $^{71}$. CIZ1 can promote tumor growth and metastasis ${ }^{70,72-74}$ and protect cells from radiation-induced DNA damage and apoptosis ${ }^{75}$. ciz $^{-/-}$mice develop neurodegenerative phenotypes during aging ${ }^{75}$; whereas overexpression of CIZ1 in cardiomyocytes protects the heart from ischemia-reperfusion injury ${ }^{76}$, a physiological condition in which cells survive caspase- 3 activation $^{9}$. CIZ1 is also overexpressed in many solid tumors ${ }^{67,77}$. All these studies suggest the role of CIZ1 in promoting survival from stress-induced executioner caspase activation may be conserved from Drosophila to mammals. Activation of PI3K-Akt signaling and oncogenic Ras are common in tumors. The fact that loss of dCIZ1 blocked survival in tissues with high level of PI3K-Akt1 activity or Ras ${ }^{\mathrm{V} 12}$, suggests it would be an excellent therapeutic target for cancer treatment. The discovery of dCIZ1 as a general regulator of survival from executioner caspase activation in development, regeneration, and oncogene-driven proliferation thus represents a significant advance.

\footnotetext{
Methods

Fly stocks. To construct the L-CasExpress sensor, DNA encoding mCD8, and amino acids 2-147 of Diap1 (mCD8-DQVD) was cloned from the previously reported CasExpress sensor ${ }^{11}$. DNA encoding lexA::p65 fusion protein was cloned from pBPLexA::p65Uw (Addgene\#26231). The $m C D 8-D Q V D$ and lexA::p65 fragments were then ligated into pattB-Ubi vector ${ }^{11}$ using In-Fusion Cloning Kit (Clontech). The construct was injected into attP2 site by BestGene Inc.

To make UAS-dCIZ1:3Xflag transgenic flies, we cloned the cDNA of dCIZ1 using clone LD27033 (Drosophila Genomics Resource Center) as the template and put it into the pJFRC7-20XUAS vector (made from Addgene\#26220). The construct was injected into attP40 site by BestGene Inc.

All the RNAi stocks used in the RNAi screening are listed in Supplementary data set 1 . The other fly stocks used in this study are listed in Supplementary Table S1. The L-trace flies were made through recombination of lexO-flp (II) and FRT-STOP-FRT-GFP (II).
} 
The genotypes of the samples shown in the figures are listed in Supplementary Table S2.

Stress and recovery assays. For all stress and recovery experiments including heat shock, radiation, and transient $r p r$ overexpression, embryos were collected in an $8 \mathrm{~h}$ window at $25^{\circ} \mathrm{C}$, and the embryo collection plates were then transferred to $18^{\circ} \mathrm{C}$. 2 days after embryo collection, larvae were collected into food vials with additional dry yeast at 50 larvae per vial. For heat shock and radiation, larvae were heat shock at $37^{\circ} \mathrm{C}$ for $2 \mathrm{~h}$ or irradiated with $10 \mathrm{~Gy} \mathrm{X}$-ray at $0.3069 \mathrm{~Gy} / \mathrm{min}$ on day 7 after egg-laying, then transferred to $29^{\circ} \mathrm{C}$ for $1 \mathrm{~d}$, followed by dissection or an additional 3 days at $18^{\circ} \mathrm{C}$. For transient $r p r$ overexpression, on day 7 after egglaying, larvae were shifted to $29^{\circ} \mathrm{C}$ for $1 \mathrm{~d}$, then the larvae were either dissected or shifted back to $18^{\circ} \mathrm{C}$ for recovery.

RNAi screening. CasExpress is activated in sperm due to the essential role of executioner caspase in sperm differentiation ${ }^{25}$. Thus CasExpress and L-trace need to be kept in separate stocks. We first made en-Gal4 UAS-RFP/CyOGFP; $L$ CasExpress/TM6B stock and en-Gal4 UAS-RFP L-trace/CyO stock for RNAi screening. Each UAS-RNAi on the third chromosome was crossed into $L$-trace background, and the L-trace/CyOGFP; UAS-RNAi/TM6B flies were then crossed to en-Gal4 UAS-RFP/CyOGFP; L-CasExpress/TM6B flies to get the en-Gal4 UAS-RFP/ $L$-trace; L-CasExpress/UAS-RNAi larvae. Each UAS-RNAi on the second chromosome was crossed into L-CasExpress background, and the UAS-RNAi/CyOGFP; $L$ CasExpress/TM6B flies were then crossed to en-Gal4 UAS-RFP L-trace/CyO flies to get en-Gal4 UAS-RFP L-trace/UAS-RNAi; L-CasExpress/+ larvae. For genes like $a k t 1$, which when knocked down at $25^{\circ} \mathrm{C}$ causes lethality, we used Gal80 ts to limit the knock-down to 3 days. The wing disc dissection, imaging, and quantification procedures are described in detail in the following sections. RNAi that caused a statistically significant change higher than $20 \%$ on CasExpress P/ A were considered as candidates for regulators. Among the candidates, only those with $\mathrm{cDcp} 1^{+}$cells changed in the direction opposite to change in CasExpress P/A were considered as regulators for survival from executioner caspase activation.

Immunostaining and imaging. Larvae at mid or late third instar were dissected and fixed in $4 \%$ paraformaldehyde (Electron Microscope Sciences) for $20 \mathrm{~min}$ at room temperature. After fixation, samples were rinsed with PBS twice and washed with PBS + 0.2\% Trition X-100 (Sigma) $(0.2 \%$ PBT) for 20 min. Samples were then blocked with $5 \%$ goat serum in $0.2 \%$ PBT for $30 \mathrm{~min}$, followed by overnight incubation with primary antibody solution at $4^{\circ} \mathrm{C}$. Next day, the samples were washed with $0.2 \%$ PBT three times, and incubated with secondary antibody and Hoechst solution (Molecular Probes) for $1 \mathrm{~h}$ at room temperature. Samples were then washed three times with $0.2 \%$ PBT and mounted on slides using Vectashield mounting medium (Vector Laboratories). The primary and secondary antibodies used in the immunostaining are listed in Supplementary Table S3. Images were taken using Zeiss LSM 780 confocal microscope and Zeiss LSM 880 confocal microscope. The software used in image capture is Zen Black (version 14.0, Zeiss).

Image analysis and quantification. All the image analysis and quantification were done using ImageJ (version 2.0). To evaluate the effect of transgene on CasExpress activation, the $\mathrm{GFP}^{+}$nuclei area and total nuclei area in both the posterior compartment $(\mathrm{P})$ and the anterior compartment $(\mathrm{A})$ was measured. Then the percentage of $\mathrm{GFP}^{+}$nuclei in each compartment was calculated, and CasExpress P/A ratio was calculated. To quantify cell death, $\mathrm{cDcp1}{ }^{+}$area and RFP area were measured and the ratio between them was calculated.

RNA extraction, reverse transcription, and qPCR. For each genotype, about 50 late third instar wing discs were dissected in PBS, and immediately transferred to TRIZOL (ThermoFisher Scientific). The total RNA was extracted using standard TRIZOL RNA extraction protocol. Turbo DNase (ThermoFisher Scientific) was used to remove genomic DNA contamination. Reverse transcription was done using SuperScript III First-Strand Synthesis System (ThermoFisher Scientific). qPCR was done using SsoAdvanced Universal SYBR Green Supermix (Bio-rad) on Bio-rad CFX96 real-time PCR detection system. The data were collected using Biorad CFX Manager software (version 3.1, Bio-rad). The primers used for qPCR were listed in Supplementary Table S4.

Transfection, protein sample preparation, co-immunoprecipitation, and western blotting. Drosophila S2 cells (Thermo Fisher Scientific) were cultured in Schneider's Drosophila medium (Thermo Fisher Scientific) supplemented with $10 \%$ fetal bovine serum (Sigma) and 100U/ml Penicillin-Streptomycin (Thermo Fisher Scientific). One day before transfection, S2 cells were seeded in 6-well plates. pMT-Gal4 plasmid and pJFRC-20XUAS-dCIZ1:3Xflag plasmid were transfected into S2 cells using Effectene Transfection Reagent (Qiagen) following the manual. Next day, $500 \mu \mathrm{M} \mathrm{CuSO}_{4}$ was added to the cells to induce Gal4 expression. After one-day induction, cells were collected and lysed in RIPA buffer (for western blotting) or TBS with $1 \%$ Trition X-100 (for co-immunoprecipitation) supplemented with $1 \mathrm{mM}$ PMSF. Co-immunoprecipitation was done using antiFLAG M2 magnetic beads (Sigma). The protein samples were applied to $10 \%$ SDSPAGE. The primary antibodies used in western blotting are listed in Supplementary Table S3. The original scans of all blots were in Source Data file.

Statistics and reproducibility. All the statistical analyses were done using Prism 6 software (GraphPad). Statistical significance was determined after logarithm transformation using unpaired two-tailed t-test for two-sample comparison or oneway ANOVA for multiple-sample comparison. The Tukey test was used to derive adjusted $P$-value for multiple comparisons. All experiments were independently repeated at least three times to ensure reproducibility, and the representative images are shown in the figures.

Reporting summary. Further information on research design is available in the Nature Research Reporting Summary linked to this article.

\section{Data availability}

All the raw data supporting the findings of this study are available from the corresponding authors upon request. Source data are provided with this paper.

Received: 9 December 2019; Accepted: 22 September 2020; Published online: 12 November 2020

\section{References}

1. Cavallucci, V. \& D’Amelio, M. In Apoptosome (eds. Cecconi, F. \& Amelio, M. D') 1-26 https://doi.org/10.1007/978-90-481-3415-1_1 (Springer Netherlands, 2010).

2. Green, D. \& Kroemer, G. The central executioners of apoptosis: caspases or mitochondria? Trends Cell Biol. 8, 267-271 (1998).

3. Sun, G. et al. A molecular signature for anastasis, recovery from the brink of apoptotic cell death. J. Cell Biol. 216, 3355-3368 (2017).

4. Tang, H. L. et al. Cell survival, DNA damage, and oncogenic transformation after a transient and reversible apoptotic response. Mol. Biol. Cell 23, 2240-2252 (2012)

5. Berthenet, K., Castillo Ferrer, C., Popgeorgiev, N., Hernandez-Vargas, H. \& Ichim, G. Failed apoptosis enhances melanoma cancer cells aggressiveness. BioRxiv https://doi.org/10.1101/755744 (2019).

6. Sun, G. \& Montell, D. J. Q\&A: Cellular near death experiences-what is anastasis? BMC Biol. 15, 92 (2017).

7. Liu, X. et al. Caspase- 3 promotes genetic instability and carcinogenesis. Mol Cell 58, 284-296 (2015).

8. Seervi, M., Sumi, S., Chandrasekharan, A., Sharma, A. K. \& SanthoshKumar, T. R. Molecular profiling of anastatic cancer cells: potential role of the nuclear export pathway. Cell Oncol. (Dordr.) 42, 645-661 (2019).

9. Kenis, H. et al. Annexin A5 uptake in ischemic myocardium: demonstration of reversible phosphatidylserine externalization and feasibility of radionuclide imaging. J. Nucl. Med. 51, 259-267 (2010).

10. de Calignon, A. et al. Caspase activation precedes and leads to tangles. Nature 464, 1201-1204 (2010).

11. Ding, A. X. et al. CasExpress reveals widespread and diverse patterns of cell survival of caspase-3 activation during development in vivo. elife 5, e10936 (2016).

12. Tang, H. L., Tang, H. M., Fung, M. C. \& Hardwick, J. M. In vivo CaspaseTracker biosensor system for detecting anastasis and non-apoptotic caspase activity. Sci. Rep. 5, 9015 (2015).

13. Pérez-Garijo, A., Martín, F. A. \& Morata, G. Caspase inhibition during apoptosis causes abnormal signalling and developmental aberrations in Drosophila. Development 131, 5591-5598 (2004).

14. Evans, C. J. et al. G-TRACE: rapid Gal4-based cell lineage analysis in Drosophila. Nat. Methods 6, 603-605 (2009).

15. Tawa, P. et al. Catalytic activity of caspase-3 is required for its degradation: stabilization of the active complex by synthetic inhibitors. Cell Death Differ. 11, 439-447 (2004).

16. White, K. et al. Genetic control of programmed cell death in Drosophila. Science 264, 677-683 (1994)

17. Yagi, R., Mayer, F. \& Basler, K. Refined LexA transactivators and their use in combination with the Drosophila Gal4 system. Proc. Natl Acad. Sci. USA 107, 16166-16171 (2010).

18. Santabárbara-Ruiz, P. et al. ROS-Induced JNK and p38 signaling is required for unpaired cytokine activation during drosophila regeneration. PLoS Genet 11, e1005595 (2015).

19. Shinoda, N., Hanawa, N., Chihara, T., Koto, A. \& Miura, M. Droncindependent basal executioner caspase activity sustains Drosophila imaginal tissue growth. Proc. Natl Acad. Sci. USA 116, 20539-20544 (2019). 
20. Florentin, A. \& Arama, E. Caspase levels and execution efficiencies determine the apoptotic potential of the cell. J. Cell Biol. 196, 513-527 (2012).

21. Baena-Lopez, L. A. et al. Novel initiator caspase reporters uncover previously unknown features of caspase-activating cells. Development 145, dev170811 (2018).

22. Siegrist, S. E., Haque, N. S., Chen, C.-H., Hay, B. A. \& Hariharan, I. K. Inactivation of both Foxo and reaper promotes long-term adult neurogenesis in Drosophila. Curr. Biol. 20, 643-648 (2010).

23. Bunker, B. D., Nellimoottil, T. T., Boileau, R. M., Classen, A. K. \& Bilder, D. The transcriptional response to tumorigenic polarity loss in Drosophila. elife 4, e03189 (2015)

24. Huh, J. R. et al. Multiple apoptotic caspase cascades are required in nonapoptotic roles for Drosophila spermatid individualization. PLoS Biol. 2, E15 (2004)

25. Arama, E., Agapite, J. \& Steller, H. Caspase activity and a specific cytochrome $\mathrm{C}$ are required for sperm differentiation in Drosophila. Dev. Cell 4, 687-697 (2003).

26. Milán, M., Campuzano, S. \& García-Bellido, A. Developmental parameters of cell death in the wing disc of Drosophila. Proc. Natl Acad. Sci. USA 94, 5691-5696 (1997).

27. Dhanasekaran, D. N. \& Reddy, E. P. JNK-signaling: a multiplexing hub in programmed cell death. Genes Cancer 8, 682-694 (2017).

28. Chatterjee, N. \& Bohmann, D. A versatile $\Phi C 31$ based reporter system for measuring AP-1 and Nrf2 signaling in Drosophila and in tissue culture. PLoS ONE 7, e34063 (2012)

29. Blanco, E. et al. Gene expression following induction of regeneration in Drosophila wing imaginal discs. Expression profile of regenerating wing discs. BMC Dev. Biol. 10, 94 (2010).

30. Zhao, B., Tumaneng, K. \& Guan, K.-L. The Hippo pathway in organ size control, tissue regeneration and stem cell self-renewal. Nat. Cell Biol. 13, 877-883 (2011).

31. Fruman, D. A. \& Rommel, C. PI3K and cancer: lessons, challenges and opportunities. Nat. Rev. Drug Disco. 13, 140-156 (2014).

32. Levayer, R., Dupont, C. \& Moreno, E. Tissue crowding induces caspasedependent competition for space. Curr. Biol. 26, 670-677 (2016).

33. Moreno, E. \& Basler, K. dMyc transforms cells into super-competitors. Cell 117, 117-129 (2004).

34. la Cova, C., de Abril, M., Bellosta, P., Gallant, P. \& Johnston, L. A. Drosophila myc regulates organ size by inducing cell competition. Cell 117, 107-116 (2004).

35. Liu, T. et al. Ciz1 promotes tumorigenicity of prostate carcinoma cells. Front Biosci. (Landmark Ed.) 20, 705-715 (2015).

36. Pérez, E., Lindblad, J. L. \& Bergmann, A. Tumor-promoting function of apoptotic caspases by an amplification loop involving ROS, macrophages and JNK in Drosophila. elife 6, e26747 (2017).

37. Prober, D. A. \& Edgar, B. A. Interactions between Ras1, dMyc, and dPI3K signaling in the developing Drosophila wing. Genes Dev. 16, 2286-2299 (2002).

38. Worley, M. I., Setiawan, L. \& Hariharan, I. K. Regeneration and transdetermination in Drosophila imaginal discs. Annu Rev. Genet 46, 289-310 (2012).

39. Fogarty, C. E. \& Bergmann, A. Killers creating new life: caspases drive apoptosis-induced proliferation in tissue repair and disease. Cell Death Differ. 24, 1390-1400 (2017).

40. Sun, G. \& Irvine, K. D. Regulation of Hippo signaling by Jun kinase signaling during compensatory cell proliferation and regeneration, and in neoplastic tumors. Dev. Biol. 350, 139-151 (2011).

41. Ryoo, H. D. \& Bergmann, A. The role of apoptosis-induced proliferation for regeneration and cancer. Cold Spring Harb. Perspect. Biol. 4, a008797 (2012).

42. Huh, J. R., Guo, M. \& Hay, B. A. Compensatory proliferation induced by cell death in the Drosophila wing disc requires activity of the apical cell death caspase Dronc in a nonapoptotic role. Curr. Biol. 14, 1262-1266 (2004).

43. Boland, K., Flanagan, L. \& Prehn, J. H. M. Paracrine control of tissue regeneration and cell proliferation by Caspase-3. Cell Death Dis. 4, e725 (2013).

44. Li, F. et al. Apoptotic cells activate the "phoenix rising" pathway to promote wound healing and tissue regeneration. Sci. Signal 3, ra13 (2010).

45. Pellettieri, J. et al. Cell death and tissue remodeling in planarian regeneration. Dev. Biol. 338, 76-85 (2010).

46. Chera, S. et al. Apoptotic cells provide an unexpected source of Wnt3 signaling to drive hydra head regeneration. Dev. Cell 17, 279-289 (2009).

47. Bonner, C. et al. INS-1 cells undergoing caspase-dependent apoptosis enhance the regenerative capacity of neighboring cells. Diabetes 59, 2799-2808 (2010).

48. Madan, E., Gogna, R. \& Moreno, E. Cell competition in development: information from flies and vertebrates. Curr. Opin. Cell Biol. 55, 150-157 (2018).
49. de Beco, S., Ziosi, M. \& Johnston, L. A. New frontiers in cell competition. Dev. Dyn. 241, 831-841 (2012).

50. Ichim, G. \& Tait, S. W. G. A fate worse than death: apoptosis as an oncogenic process. Nat. Rev. Cancer 16, 539-548 (2016).

51. Zhao, R. et al. Novel roles of apoptotic caspases in tumor repopulation, epigenetic reprogramming, carcinogenesis, and beyond. Cancer Metastasis Rev. 37, 227-236 (2018)

52. Yang, X. et al. Caspase-3 over-expression is associated with poor overall survival and clinicopathological parameters in breast cancer: a meta-analysis of 3091 cases. Oncotarget 9, 8629-8641 (2018).

53. Zhou, M. et al. Caspase- 3 regulates the migration, invasion and metastasis of colon cancer cells. Int J. Cancer 143, 921-930 (2018).

54. Donato, A. L. et al. Caspase 3 promotes surviving melanoma tumor cell growth after cytotoxic therapy. J. Invest Dermatol 134, 1686-1692 (2014).

55. Feng, X. et al. Caspase 3 in dying tumor cells mediates post-irradiation angiogenesis. Oncotarget 6, 32353-32367 (2015).

56. Huang, Q. et al. Caspase 3-mediated stimulation of tumor cell repopulation during cancer radiotherapy. Nat. Med 17, 860-866 (2011).

57. Ford, C. A. et al. Oncogenic properties of apoptotic tumor cells in aggressive B cell lymphoma. Curr. Biol. 25, 577-588 (2015).

58. Cheng, J. et al. Dying tumor cells stimulate proliferation of living tumor cells via caspase-dependent protein kinase $C \delta$ activation in pancreatic ductal adenocarcinoma. Mol. Oncol. 9, 105-114 (2015).

59. Manning, B. D. \& Toker, A. AKT/PKB signaling: navigating the network. Cell 169, 381-405 (2017).

60. Manning, B. D. \& Cantley, L. C. AKT/PKB signaling: navigating downstream. Cell 129, 1261-1274 (2007)

61. Zhou, H., Li, X. M., Meinkoth, J. \& Pittman, R. N. Akt regulates cell survival and apoptosis at a postmitochondrial level. J. Cell Biol. 151, 483-494 (2000).

62. Khalil, H. et al. Caspase- 3 protects stressed organs against cell death. Mol. Cell Biol. 32, 4523-4533 (2012).

63. Belozerov, V. E., Lin, Z.-Y., Gingras, A.-C., McDermott, J. C. \& Michael Siu, K. W. High-resolution protein interaction map of the Drosophila melanogaster p38 mitogen-activated protein kinases reveals limited functional redundancy. Mol. Cell Biol. 32, 3695-3706 (2012).

64. Breitkopf, S. B. et al. A cross-species study of PI3K protein-protein interactions reveals the direct interaction of P85 and SHP2. Sci. Rep. 6, 20471 (2016).

65. Mitsui, K., Matsumoto, A., Ohtsuka, S., Ohtsubo, M. \& Yoshimura, A. Cloning and characterization of a novel p21(Cip1/Waf1)-interacting zinc finger protein, ciz1. Biochem Biophys. Res Commun. 264, 457-464 (1999).

66. Coverley, D., Marr, J. \& Ainscough, J. Cizl promotes mammalian DNA replication. J. Cell Sci. 118, 101-112 (2005).

67. Pauzaite, T., Thacker, U., Tollitt, J. \& Copeland, N. A. Emerging roles for ciz1 in cell cycle regulation and as a driver of tumorigenesis. Biomolecules 7, https://doi.org/10.3390/biom7010001 (2016)

68. Hollander, P., den, Rayala, S. K., Coverley, D. \& Kumar, R. Ciz1, a Novel DNA-binding coactivator of the estrogen receptor alpha, confers hypersensitivity to estrogen action. Cancer Res. 66, 11021-11029 (2006).

69. Lei, L., Wu, J., Gu, D., Liu, H. \& Wang, S. CIZ1 interacts with YAP and activates its transcriptional activity in hepatocellular carcinoma cells. Tumour Biol. 37, 11073-11079 (2016)

70. Zhang, D. et al. CIZ1 promoted the growth and migration of gallbladder cancer cells. Tumour Biol. 36, 2583-2591 (2015).

71. Stewart, E. R. et al. Maintenance of epigenetic landscape requires CIZ1 and is corrupted in differentiated fibroblasts in long-term culture. Nat. Commun. 10, 460 (2019).

72. Wu, J., Lei, L., Gu, D., Liu, H. \& Wang, S. CIZ1 is upregulated in hepatocellular carcinoma and promotes the growth and migration of the cancer cells. Tumour Biol. 37, 4735-4742 (2016).

73. Yin, J. et al. CIZ1 regulates the proliferation, cycle distribution and colony formation of RKO human colorectal cancer cells. Mol. Med Rep. 8, 1630-1634 (2013).

74. Chen, X. et al. CIZ1 knockdown suppresses the proliferation of bladder cancer cells by inducing apoptosis. Gene 143946 https://doi.org/10.1016/j. gene.2019.143946 (2019).

75. Khan, M. M., Xiao, J., Patel, D. \& LeDoux, M. S. DNA damage and neurodegenerative phenotypes in aged Cizl null mice. Neurobiol. Aging 62, 180-190 (2018)

76. Bageghni, S. A. et al. Cardiomyocyte-specific expression of the nuclear matrix protein, CIZ1, stimulates production of mono-nucleated cells with an extended window of proliferation in the postnatal mouse heart. Biol. Open 6 92-99 (2017).

77. Liu, Q., Niu, N., Wada, Y. \& Liu, J. The Role of Cdkn1A-Interacting Zinc Finger Protein 1 (CIZ1) in DNA replication and pathophysiology. Int J. Mol. Sci. 17, 212 (2016). 


\section{Acknowledgments}

We thank Dr. David Bilder, Dr. Florenci Serras, Dr. Kristin White, Dr. Alfonso MartinezArias, Dr. Rachel K. Smith-Bolton, Bloomington Stock Center, and Vienna Drosophila Research Center for providing fly stocks. We thank James Mondo for the FIJI macro used for cDcpl staining quantification. We thank Rebecca Cheng, Marni Nashimoto, Yufeng Wang, Xiaojiao Li, and Advanced Medical Research Institute, Shandong University for technical support. This work was supported by NIH grant DP1OD019313 to D.J.M.

\section{Author contributions}

G.S. and D.J.M. designed the experiments. G.S., A.X.D., Y.A., and X.G. carried out the experiments. G.S. and D.J.M. prepared the manuscript.

\section{Competing interests}

The authors declare no competing interests.

\section{Additional information}

Supplementary information is available for this paper at https://doi.org/10.1038/s41467020-19068-2.

Correspondence and requests for materials should be addressed to G.S. or D.J.M.
Peer review information Nature Communications thanks the anonymous reviewer(s) for their contribution to the peer review of this work.

Reprints and permission information is available at http://www.nature.com/reprints

Publisher's note Springer Nature remains neutral with regard to jurisdictional claims in published maps and institutional affiliations.

(c) (i) Open Access This article is licensed under a Creative Commons Attribution 4.0 International License, which permits use, sharing, adaptation, distribution and reproduction in any medium or format, as long as you give appropriate credit to the original author(s) and the source, provide a link to the Creative Commons license, and indicate if changes were made. The images or other third party material in this article are included in the article's Creative Commons license, unless indicated otherwise in a credit line to the material. If material is not included in the article's Creative Commons license and your intended use is not permitted by statutory regulation or exceeds the permitted use, you will need to obtain permission directly from the copyright holder. To view a copy of this license, visit http://creativecommons.org/ licenses/by/4.0/.

(C) The Author(s) 2020 